\title{
Time-Dependent Variational Inequality for an Oligopolistic Market Equilibrium Problem with Production and Demand Excesses
}

\author{
Annamaria Barbagallo ${ }^{1}$ and Paolo Mauro ${ }^{2}$ \\ ${ }^{1}$ Department of Mathematics and Applications "R. Caccioppoli", University of Naples "Federico II", \\ Via Cintia 80126 Naples, Italy \\ ${ }^{2}$ Department of Mathematics and Computer Science, University of Catania, \\ Viale A. Doria, 95125 Catania, Italy
}

Correspondence should be addressed to Annamaria Barbagallo, annamaria.barbagallo@unina.it

Received 2 February 2012; Accepted 20 March 2012

Academic Editor: Kanishka Perera

Copyright @ 2012 A. Barbagallo and P. Mauro. This is an open access article distributed under the Creative Commons Attribution License, which permits unrestricted use, distribution, and reproduction in any medium, provided the original work is properly cited.

The paper is concerned with the variational formulation of the oligopolistic market equilibrium problem in presence of both production and demand excesses. In particular, we generalize a previous model in which the authors, instead, considered only the problem with production excesses, by allowing also the presence of demand excesses. First we examine the equilibrium conditions in terms of the well-known dynamic Cournot-Nash principle. Next, the equilibrium conditions will be expressed in terms of Lagrange multipliers by means of the infinite dimensional duality theory. Then, we show the equivalence between the two conditions that are both expressed by an appropriate evolutionary variational inequality. Moreover, thanks to the variational formulation, some existence and regularity results for equilibrium solutions are proved. At last, a numerical example, which illustrates the features of the problem, is provided.

\section{Introduction}

The aim of this paper is to introduce a time-dependent variational formulation for the dynamic oligopolistic market equilibrium model in presence of both production and demand excesses. Moreover, in line with [1], we want to eliminate the serious drawback present in [2] where the authors made the unreasonable assumption that the production of a given commodity could be unbounded, by making possible any commodity shipment from a firm to a demand market. This is not possible because the amount of a commodity that the producers can offer is limited as a consequence of finite resources. Therefore, it can happen that some of the amounts of the available commodity are sold out so that can occur 
an excess of demand, whereas for a part of the producers can occur an excess of production. The question about the unbounded production had already been solved in [1], but the presence of both excesses realizes a more complete study of the problem. In order to clarify the presence of both production and demand excesses we consider some concrete economic situations. During an economic crisis period the presence of production excesses can be due to a demand decrease in demand markets and, on the other hand, the presence of demand excesses may occur when the supply cannot satisfy the demand especially for fundamental goods. Moreover, since the market model presented in this paper evolves in time, the presence of both production and demand excesses is a consequence of the fact that the physical transportation of commodity between a firm and a demand market is evidently limited, therefore, there can exist some time intervals in which some of the demand markets require more commodity, though some firms produce more commodity than they can send to all the demand markets. For these reasons the new model results in a more realistic generalization of the ones presented in $[1,2]$, where the authors studied the case without presence of excesses and the case with the only presence of production excesses, respectively.

The equilibrium formulation fits in the light of a dynamic noncooperative behaviour. The first author who treated the most trivial example of noncooperative behaviour between two producers of a given commodity, nowadays called duopoly problem, was Cournot (see [3]). Later Nash, in [4,5], extended this concept by introducing $m$ agents in his model nowadays called noncooperative game, each acting according to his own self-interest.

In order to study the time-dependent behaviour of the model, we will afford this study by considering the evolution of the market in time and, as a consequence, all the variables present in this model, such as the costs, the commodity shipments, and the excesses depend on time. As Beckmann and Wallace pointed out, for the first time, in [6], "the time-dependent formulation of equilibrium problems allows one to explore the dynamics of adjustment processes in which a delay on time response is operating." Of course a delay on time response always happens because the processes have not an infinite speed. Usually, such adjustment processes can be represented by means of a memory term which depends on previous equilibrium solutions according to the Volterra operator (see, e.g., [7]). The time-dependent process was one of the main features of the paper [2] where, in particular, the authors studied the variational formulation and proved the existence and regularity of a dynamic equilibrium solution. Moreover, the regularity property allows to provide a computational procedure to compute the equilibrium solutions (see, e.g., [8-12]).

In [13] the authors, through the notion of quasi-relative interior of sets (see [14]), applied the infinite dimensional duality results developed in [15-18] to overcome the difficulty of the voidness of the interior of the ordering cone which defines the constraints of the problem and so proved the existence of Lagrange variables which permit to describe the behaviour of the market. Moreover, in [13] some sensitivity results have been obtained each of them showing that small changes of the solution happen in correspondence with small changes of the profit function. In [7, 19], an oligopolistic market equilibrium model with an explicit long-term memory has been considered. Then, in [19], the Lipschitz continuity of the solution, which depends on the variation rate of projections onto time-dependent constraints set, is shown and the existence of Lagrange multipliers is provided.

In the first part of the paper, we present the equilibrium conditions in presence of excesses according to the well-known Cournot-Nash principle. This is a practical equilibrium definition that expresses that each firm acts trying to maximize its own profit. The second one, more theoretical, is shown through the Lagrange variables that better emphasize, in particular, the presence of excess constraints. The equilibrium conditions established in terms 
of Lagrange variables do not arouse any concern because we will prove that their presence is not influential in the definition of equilibrium because we can characterize such equilibrium conditions by means of an evolutionary variational inequality that does not contain the Lagrange variables. Another thing to notice is that the equilibrium conditions, provided with the help of the duality theory, are equivalent to the dynamic Cournot-Nash equilibrium principle because we can prove that they are both equivalent to the same evolutionary variational inequality. Such variational formulation gives us a powerful tool for the study of the existence, the regularity, and the calculus of equilibrium solutions. In particular, we show that the constraint set satisfies the property of set convergence in Kuratowski's sense which has an important role in order to guarantee the continuity of equilibrium solutions. Moreover, the continuity property is very useful in order to introduce a numerical scheme to compute equilibrium solutions (see [20,21]).

The outline of the paper is as the following. In Section 2, we describe the model of the dynamic oligopolistic market equilibrium problem in presence of both production and demand excesses and we show the equilibrium conditions making use of both CournotNash principle and Lagrange multipliers. In Section 3, we recall the new infinite dimensional duality theory requested to show the existence of Lagrange variables. In Section 4, after showing some preliminary lemmas, we give the proof of the characterization of the dynamic oligopolistic market equilibrium conditions established in terms of Lagrange variables by means of an evolutionary variational inequality, so we can derive their equivalence with the dynamic Cournot-Nash principle. In Section 5, after recalling some preliminary definitions, we give some existence results. Section 6 is devoted to provide a regularity result for the equilibrium solution after proving that the constraint set of commodity shipments satisfies the requirements of the set convergence in Kuratowski's sense. Finally, in Section 7, we provide a numerical example of a dynamic oligopolistic market equilibrium problem in presence of production and demand excesses and underline some important features of the problem.

\section{Dynamic Oligopolistic Market Equilibrium}

Let us consider $m$ firms $P_{i}, i=1, \ldots, m$, that produce only one commodity and $n$ demand markets $Q_{j}, j=1, \ldots, n$, that are generally spatially separated. Assume that the homogeneous commodity, produced by the $m$ firms and consumed by the $n$ markets, is involved during a time interval $[0, T], T>0$. Let $p_{i}(t), i=1, \ldots, m$, denote the nonnegative commodity output produced by firm $P_{i}$ at the time $t \in[0, T]$. Let $q_{j}(t), j=1, \ldots, n$, denote the nonnegative demand for the commodity at demand market $Q_{j}$ at the time $t \in[0, T]$. Let $x_{i j}(t), i=1, \ldots, m$, $j=1, \ldots, n$, denote the nonnegative commodity shipment between the supply market $P_{i}$ and the demand market $Q_{j}$ at the time $t \in[0, T]$. In particular, let us set the vector $x_{i}(t)=$ $\left(x_{i 1}(t), \ldots, x_{i n}(t)\right), i=1, \ldots, m, t \in[0, T]$ as the strategy vector for the firm $P_{i}$. Finally, let us introduce the production and demand excesses. Let $\epsilon_{i}(t), i=1, \ldots, m$, be the nonnegative production excess for the commodity of the firm $P_{i}$ at the time $t \in[0, T]$. Let $\delta_{j}(t), j=1, \ldots, n$, be the nonnegative demand excess for the commodity of the demand market $Q_{j}$ at the time $t \in[0, T]$.

Let us group the production output into a vector-function $p:[0, T] \rightarrow \mathbb{R}_{+}^{m}$, the demand output into a vector-function $q:[0, T] \rightarrow \mathbb{R}_{+}^{n}$, the commodity shipments into a matrix-function $x:[0, T] \rightarrow \mathbb{R}_{+}^{m n}$, the production excess into a vector-function $\epsilon:[0, T] \rightarrow$ $\mathbb{R}_{+}^{m}$, and the demand excess into a vector-function $\delta:[0, T] \rightarrow \mathbb{R}_{+}^{n}$. 
Let us assume that the following feasibility conditions hold:

$$
\begin{aligned}
& p_{i}(t)=\sum_{j=1}^{n} x_{i j}(t)+\epsilon_{i}(t), \quad i=1, \ldots, m, \text { a.e. in }[0, T], \\
& q_{j}(t)=\sum_{i=1}^{m} x_{i j}(t)+\delta_{j}(t), \quad j=1, \ldots, n, \text { a.e. in }[0, T] .
\end{aligned}
$$

Hence, the quantity produced by each firm $P_{i}$, at the time $t \in[0, T]$, must be equal to the commodity shipments from that firm to all the demand markets plus the production excess, at the same time $t \in[0, T]$. Moreover, the quantity demanded by each demand market $Q_{j}$, at the time $t \in[0, T]$, must be equal to the commodity shipments from all the firms to that demand market plus the demand excess, at the same time $t \in[0, T]$.

Furthermore, we assume that the nonnegative commodity shipment between the producer $P_{i}$ and the demand market $Q_{j}$ has to satisfy time-dependent constraints, namely, there exist two nonnegative functions $\underline{x}, \bar{x}:[0, T] \rightarrow \mathbb{R}_{+}^{m n}$ such that

$$
0 \leq \underline{x}_{i j}(t) \leq x_{i j}(t) \leq \bar{x}_{i j}(t), \quad \forall i=1, \ldots, m, \forall j=1, \ldots, n \text {, a.e. in }[0, T] .
$$

For technical reasons, let us assume that

$$
\begin{gathered}
x \in L^{2}\left([0, T], \mathbb{R}_{+}^{m n}\right), \quad \underline{x} \in L^{2}\left([0, T], \mathbb{R}_{+}^{m n}\right), \quad \bar{x} \in L^{2}\left([0, T], \mathbb{R}_{+}^{m n}\right), \\
\epsilon \in L^{2}\left([0, T], \mathbb{R}_{+}^{m}\right), \quad \delta \in L^{2}\left([0, T], \mathbb{R}_{+}^{n}\right) .
\end{gathered}
$$

As a consequence, we have

$$
p \in L^{2}\left([0, T], \mathbb{R}_{+}^{m}\right), \quad q \in L^{2}\left([0, T], \mathbb{R}_{+}^{n}\right) .
$$

Then, the set of feasible vectors $(x, \epsilon, \delta) \in L^{2}\left([0, T], \mathbb{R}_{+}^{m n+m+n}\right)$ is

$$
\begin{aligned}
\mathbb{K}^{*}=\left\{(x, \epsilon, \delta) \in L^{2}\left([0, T], \mathbb{R}_{+}^{m n+m+n}\right):\right. \\
\quad \underline{x}_{i j}(t) \leq x_{i j}(t) \leq \bar{x}_{i j}(t), \forall i=1, \ldots, m, \forall j=1, \ldots, n, \text { a.e. in }[0, T], \\
\epsilon_{i}(t) \geq 0, \forall i=1, \ldots, m, \text { a.e. in }[0, T], \\
p_{i}(t)=\sum_{j=1}^{n} x_{i j}(t)+\epsilon_{i}(t), \forall i=1, \ldots, m, \text { a.e. in }[0, T], \\
\delta_{j}(t) \geq 0, \forall j=1, \ldots, n, \text { a.e. in }[0, T], \\
\left.q_{j}(t)=\sum_{i=1}^{m} x_{i j}(t)+\delta_{j}(t), \forall j=1, \ldots, n, \text { a.e. in }[0, T]\right\} .
\end{aligned}
$$


Furthermore, let us associate with each firm $P_{i}$ a production cost $f_{i}^{*}, i=1, \ldots, m$, and assume that the production cost of a firm $P_{i}$ may depend upon the entire production pattern, namely:

$$
f_{i}^{*}=f_{i}^{*}(t, x(t), \epsilon(t))
$$

Similarly, let us associate with each demand market $Q_{j}$, a demand price for unity of the commodity $d_{j}^{*}, j=1, \ldots, n$ and assume that the demand price of a demand market $Q_{j}$ may depend upon the entire consumption pattern, namely:

$$
d_{j}^{*}=d_{j}^{*}(t, x(t), \delta(t))
$$

Moreover, since we allow production excesses and, consequently, the storage of commodities, we must consider the function $g_{i}^{*}, i=1, \ldots, m$, that denotes the storage cost of the commodity produced by the firm $P_{i}$ and assume that this cost may depend upon the entire production pattern, namely:

$$
g_{i}^{*}=g_{i}^{*}(t, x(t), \epsilon(t))
$$

Finally, let $c_{i j}, i=1, \ldots, m, j=1, \ldots, n$, denote the transaction cost, which includes the transportation cost associated with trading the commodity between firm $P_{i}$ and demand market $Q_{j}$. Here we permit the transaction cost to depend upon the entire shipment pattern, namely:

$$
c_{i j}(t)=c_{i j}(t, x(t)) .
$$

Hence, we have the following mappings:

$$
\begin{aligned}
& f^{*}:[0, T] \times L^{2}\left([0, T], \mathbb{R}_{+}^{m n}\right) \times L^{2}\left([0, T], \mathbb{R}_{+}^{m}\right) \longrightarrow L^{2}\left([0, T], \mathbb{R}_{+}^{m}\right), \\
& d^{*}:[0, T] \times L^{2}\left([0, T], \mathbb{R}_{+}^{m n}\right) \times L^{2}\left([0, T], \mathbb{R}_{+}^{n}\right) \longrightarrow L^{2}\left([0, T], \mathbb{R}_{+}^{n}\right), \\
& g^{*}:[0, T] \times L^{2}\left([0, T], \mathbb{R}_{+}^{m n}\right) \times L^{2}\left([0, T], \mathbb{R}_{+}^{m}\right) \longrightarrow L^{2}\left([0, T], \mathbb{R}_{+}^{m}\right), \\
& \quad c:[0, T] \times L^{2}\left([0, T], \mathbb{R}_{+}^{m n}\right) \longrightarrow L^{2}\left([0, T], \mathbb{R}_{+}^{m n}\right) .
\end{aligned}
$$

The profit $v_{i}^{*}(t, x(t), \epsilon(t), \delta(t)), i=1, \ldots, m$, of the firm $P_{i}$ at the time $t \in[0, T]$ is, then,

$$
\begin{aligned}
v_{i}^{*}(t, x(t), \epsilon(t), \delta(t))= & \sum_{j=1}^{n} d_{j}^{*}(t, x(t), \delta(t)) x_{i j}(t)-f_{i}^{*}(t, x(t), \epsilon(t)) \\
& -g_{i}^{*}(t, x(t), \epsilon(t))-\sum_{j=1}^{n} c_{i j}(t, x(t)) x_{i j}(t),
\end{aligned}
$$


namely, it is equal to the price that the demand markets are disposed to pay minus the production cost, the storage cost and the transportation costs.

Now, we can rewrite $\mathbb{K}^{*}$ in an equivalent way. By virtue of (2.1) we can express $\epsilon_{i}(t)$ in terms of $p_{i}(t)$ and $x_{i j}(t)$ and $\delta_{j}(t)$ in terms of $q_{j}(t)$ and $x_{i j}(t)$, namely:

$$
\begin{aligned}
& \epsilon_{i}(t)=p_{i}(t)-\sum_{j=1}^{n} x_{i j}(t), \quad i=1, \ldots, m, \text { a.e. in }[0, T], \\
& \delta_{j}(t)=q_{j}(t)-\sum_{i=1}^{m} x_{i j}(t), \quad j=1, \ldots, n, \text { a.e. in }[0, T] .
\end{aligned}
$$

Then, the equivalent constraint set becomes

$$
\begin{aligned}
& \mathbb{K}=\left\{x \in L^{2}\left([0, T], \mathbb{R}_{+}^{m n}\right):\right. \\
& \quad \underline{x}_{i j}(t) \leq x_{i j}(t) \leq \bar{x}_{i j}(t), \forall i=1, \ldots, m, \forall j=1, \ldots, n, \text { a.e. in }[0, T], \\
& \sum_{j=1}^{n} x_{i j}(t) \leq p_{i}(t), \forall i=1, \ldots, m, \text { a.e. in }[0, T], \\
&\left.\sum_{i=1}^{m} x_{i j}(t) \leq q_{j}(t), \forall j=1, \ldots, n, \text { a.e. in }[0, T]\right\} .
\end{aligned}
$$

We can observe that $\mathbb{K}$ includes the presence of both production and demand excesses described in $\mathbb{K}^{*}$.

Then, the production costs, the demand price, and the storage costs, by virtue of (2.12) and taking into account (2.6), (2.7), and (2.8), become

$$
\begin{aligned}
& f_{i}=f_{i}(t, x(t))=f_{i}^{*}(t, x(t), \epsilon(t)), \\
& d_{j}=d_{j}(t, x(t))=d_{j}^{*}(t, x(t), \delta(t)), \\
& g_{i}=g_{i}(t, x(t))=g_{i}^{*}(t, x(t), \epsilon(t)),
\end{aligned}
$$

and, analogously, the profit function (2.11) becomes

$$
\begin{aligned}
v_{i}(t, x(t)) & =v_{i}^{*}(t, x(t), \epsilon(t), \delta(t)) \\
& =\sum_{j=1}^{n} d_{j}(t, x(t)) x_{i j}(t)-f_{i}(t, x(t))-g_{i}(t, x(t))-\sum_{j=1}^{n} c_{i j}(t, x(t)) x_{i j}(t) .
\end{aligned}
$$



tions:

Let us denote by $\nabla_{D} v=\left(\partial v_{i} / \partial x_{i j}\right)_{\substack{i=1, \ldots, m, j=1, \ldots, n}}$ and let us assume the following assump-

(i) $v_{i}(t, x(t))$ is continuously differentiable for each $i=1, \ldots, m$, a.e. in $[0, T]$,

(ii) $\nabla_{D} v$ is a Carathéodory function such that

$$
\exists h \in L^{2}([0, T]):\left\|\nabla_{D} v(t, x(t))\right\|_{m n} \leq h(t)\|x(t)\|_{m n}, \quad \text { a.e. in }[0, T],
$$

(iii) $v_{i}(t, x(t))$ is pseudoconcave with respect to the variable $x_{i}, i=1, \ldots, m$, a.e. in $[0, T]$.

For the reader's convenience, we recall that a function $v$, continuously differentiable, is called pseudoconcave with respect to $x_{i}, i=1, \ldots, m$, a.e. in [0,T] (see [22]), if the following condition holds, a.e. in $[0, T]$ :

$$
\begin{aligned}
& \left\langle\frac{\partial v}{\partial x_{i}}\left(t, x_{1}, \ldots, x_{i}, \ldots, x_{m}\right), x_{i}-y_{i}\right\rangle \geq 0 \\
& \quad \Longrightarrow v_{i}\left(t, x_{1}, \ldots, x_{i}, \ldots, x_{m}\right) \geq v_{i}\left(t, x_{1}, \ldots, y_{i}, \ldots, x_{m}\right)
\end{aligned}
$$

Now let us consider the dynamic oligopolistic market, in which the $m$ firms supply the commodity in a noncooperative fashion, each one trying to maximize its own profit function considered the optimal distribution pattern for the other firms, at the time $t \in[0, T]$. We seek to determine a nonnegative commodity distribution matrix-function $x$ for which the $m$ firms and the $n$ demand markets will be in a state of equilibrium as defined below. In fact, we can consider different, but equivalent, equilibrium conditions each of them illustrates important features of the equilibrium.

The first one makes use of the dynamic Cournot-Nash principle (see [2]).

Definition 2.1. $x^{*} \in \mathbb{K}$ is a dynamic oligopolistic market equilibrium in presence of excesses if and only if for each $i=1, \ldots, m$ and a.e. in $[0, T]$ one has

$$
v_{i}\left(t, x^{*}(t)\right) \geq v_{i}\left(t, x_{i}(t), \hat{x}_{i}^{*}(t)\right),
$$

where

$$
\widehat{x}_{i}^{*}(t)=\left(x_{1}^{*}(t), \ldots, x_{i-1}^{*}(t), x_{i+1}^{*}(t), \ldots, x_{m}^{*}(t)\right) .
$$

With the same technique used in [2, Theorem 3.1] it is possible to prove that under the assumptions (i), (ii), (iii) on $v_{i}$, Definition 2.1 is equivalent to an evolutionary variational inequality, as the following result shows.

Theorem 2.2. Let one suppose that assumptions (i), (ii), (iii) are satisfied. Then, $x^{*} \in \mathbb{K}$ is a dynamic oligopolistic market equilibrium in presence of excesses according to Definition 2.1 if and only if it satisfies the evolutionary variational inequality

$$
\int_{0}^{T}-\sum_{i=1}^{m} \sum_{j=1}^{n} \frac{\partial v_{i}\left(t, x^{*}(t)\right)}{\partial x_{i j}}\left(x_{i j}(t)-x_{i j}^{*}(t)\right) d t \geq 0, \quad \forall x \in \mathbb{K}
$$


In Section 4 we will prove that, under the assumptions (i), (ii), (iii) on the profit function $v$, Definition 2.1 is equivalent to the equilibrium conditions defined through Lagrange variables which are very useful in order to analyze the presence of both production and demand excesses.

Definition 2.3. $x^{*} \in \mathbb{K}$ is a dynamic oligopolistic market problem equilibrium in presence of excesses if and only if, for each $i=1, \ldots, m, j=1, \ldots, n$ and a.e. in $[0, T]$, there exist $\lambda_{i j}^{*} \in L^{2}([0, T]), \rho_{i j}^{*} \in L^{2}([0, T]), \mu_{i}^{*} \in L^{2}([0, T]), v_{j}^{*}(t) \in L^{2}([0, T])$ such that

$$
\begin{gathered}
-\frac{\partial v_{i}\left(t, x^{*}(t)\right)}{\partial x_{i j}}+\rho_{i j}^{*}(t)+\mu_{i}^{*}(t)+v_{j}^{*}(t)=\lambda_{i j}^{*}(t), \\
\lambda_{i j}^{*}(t)\left(\underline{x}_{i j}(t)-x_{i j}^{*}(t)\right)=0, \quad \lambda_{i j}^{*}(t) \geq 0, \\
\rho_{i j}^{*}(t)\left(x_{i j}^{*}(t)-\bar{x}_{i j}(t)\right)=0, \quad \rho_{i j}^{*}(t) \geq 0, \\
\mu_{i}^{*}(t)\left(\sum_{j=1}^{n} x_{i j}^{*}(t)-p_{i}(t)\right)=0, \quad \mu_{i}^{*}(t) \geq 0, \\
v_{j}^{*}(t)\left(\sum_{i=1}^{m} x_{i j}^{*}(t)-q_{j}(t)\right)=0, \quad v_{j}^{*}(t) \geq 0 .
\end{gathered}
$$

The terms $\lambda_{i j}^{*}(t), \rho_{i j}^{*}(t), \mu_{i}^{*}(t), v_{j}^{*}(t)$ are the Lagrange multipliers associated to the constraints $x_{i j}^{*}(t) \geq \underline{x}_{i j}(t), x_{i j}^{*}(t) \leq \bar{x}_{i j}(t), \sum_{j=1}^{n} x_{i j}^{*}(t) \leq p_{i}(t)$, and $\sum_{i=1}^{m} x_{i j}^{*}(t) \leq q_{j}(t)$, respectively.

They, as it is well known, have a topical importance on the understanding and the management of the market. In fact, at a fixed time $t \in[0, T]$, we have:

(a) if $\lambda_{i j}^{*}(t)>0$ then, by using (2.22), we obtain $x_{i j}^{*}(t)=\underline{x}_{i j}(t)$, namely, the commodity shipment between the firm $P_{i}$ and the demand market $Q_{j}$ is minimum,

(b) if $x_{i j}^{*}(t)>\underline{x}_{i j}(t)$ then, taking into account (2.22), $\lambda_{i j}^{*}(t)=0$ and, making use of (2.21), it results $\rho_{i j}^{*}(t)+\mu_{i}^{*}(t)+v_{j}^{*}(t)=\partial v_{i}\left(t, x^{*}(t)\right) / \partial x_{i j}$, namely, $\rho_{i j}^{*}(t)+\mu_{i}^{*}(t)+v_{j}^{*}(t)$ is equal to the marginal profit,

(c) if $\rho_{i j}^{*}(t)>0$ then, by using (2.23), we obtain $x_{i j}^{*}(t)=\bar{x}_{i j}(t)$, namely, the commodity shipment between the firm $P_{i}$ and the demand market $Q_{j}$ is maximum,

(d) if $x_{i j}^{*}(t)<\bar{x}_{i j}(t)$ then, making use of (2.23), $\rho_{i j}^{*}(t)=0$ and, taking into account (2.21), we get $\mu_{i}^{*}(t)+v_{j}^{*}(t)-\lambda_{i j}^{*}(t)=\partial v_{i}\left(t, x^{*}(t)\right) / \partial x_{i j}$, namely, $\mu_{i}^{*}(t)+v_{j}^{*}(t)-\lambda_{i j}^{*}(t)$ is equal to the marginal profit, 
(e) if $\mu_{i}^{*}(t)>0$ then, for the condition (2.24), we have $\sum_{j=1}^{n} x_{i j}^{*}(t)=p_{i}(t)$, namely, there is no production excess,

(f) if $\sum_{j=1}^{n} x_{i j}^{*}(t)<p_{i}(t)$, as a consequence of (2.24) we get $\mu_{i}^{*}(t)=0$ and, for the condition (2.21), $\rho_{i j}^{*}(t)+v_{j}^{*}(t)-\lambda_{i j}^{*}(t)=\partial v_{i}\left(t, x^{*}(t)\right) / \partial x_{i j}$, namely, $\rho_{i j}^{*}(t)+v_{j}^{*}(t)-\lambda_{i j}^{*}(t)$ is equal to the marginal profit,

(g) if $v_{j}^{*}(t)>0$ then, for the condition (2.25), it results $\sum_{i=1}^{m} x_{i j}^{*}(t)=q_{j}(t)$, namely, there is no demand excess,

(h) if $\sum_{i=1}^{m} x_{i j}^{*}(t)<q_{j}(t)$, as a consequence of (2.25) we obtain $v_{j}^{*}(t)=0$ and, for the condition (2.21), $\rho_{i j}^{*}(t)+\mu_{i}^{*}(t)-\lambda_{i j}^{*}(t)=\partial v_{i}\left(t, x^{*}(t)\right) / \partial x_{i j}$, namely, $\rho_{i j}^{*}(t)+\mu_{i}^{*}(t)-\lambda_{i j}^{*}(t)$ is equal to the marginal profit.

It is worthy to underline that in Definition 2.3, even if in (2.21)-(2.25) the unknown Lagrange variables $\lambda_{i j}^{*}, \rho_{i j}^{*}, \mu_{i}^{*}, v_{j}^{*}$ appear, they do not influence the equilibrium definition because the following equivalent condition in terms of evolutionary variational inequality holds.

Theorem 2.4. $x^{*} \in \mathbb{K}$ is a dynamic oligopolistic market equilibrium in presence of excesses according to Definition 2.3 if and only if it satisfies the evolutionary variational inequality:

$$
\int_{0}^{T}-\sum_{i=1}^{m} \sum_{j=1}^{n} \frac{\partial v_{i}\left(t, x^{*}(t)\right)}{\partial x_{i j}}\left(x_{i j}(t)-x_{i j}^{*}(t)\right) d t \geq 0, \quad \forall x \in \mathbb{K} .
$$

Taking into account Theorems 2.2 and 2.4, the equivalence between Definitions 2.1 and 2.3 is proved.

Finally, we observe that also in the case in which the production is bounded and we are in presence of excesses, the meaning of Cournot-Nash equilibrium does not change.

\section{Lagrange Theory}

Let us present the infinite dimensional Lagrange duality theory which represents an important and very recent achievement (see [16-18]). At first, we remember some definitions and then we give some duality results (see [15-17]).

Let $X$ denote a real normed space, let $X^{*}$ be the topological dual of all continuous linear functionals on $X$, and let $C$ be a subset of $X$. Given an element $x \in \mathrm{Cl}(C)$, the set:

$$
T_{C}(x)=\left\{h \in X: h=\lim _{n \rightarrow \infty} \lambda_{n}\left(x_{n}-x\right), \lambda_{n}>0, x_{n} \in C, \forall n \in \mathbb{N}, \lim _{n \rightarrow \infty} x_{n}=x\right\}
$$

is called the tangent cone to $C$ at $x$.

If $C$ is convex, we have (see [23]):

$$
T_{C}(x)=\mathrm{Cl}(\text { Cone }(C-\{x\})),
$$

where Cone $(C)=\{\lambda x: x \in C, \lambda \geq 0\}$. 
Following Borwein and Lewis [14], we give the following definition of quasi-relative interior for a convex set.

Definition 3.1. Let $C$ be a convex subset of $X$. The quasi-relative interior of $C$, denoted by qri $C$, is the set of those $x \in C$ for which $T_{C}(x)$ is a linear subspace of $X$.

If we define the normal cone to $C$ at $x$ as the set:

$$
N_{C}(x)=\left\{\xi \in X^{*}:\langle\xi, y-x\rangle \leq 0, \forall y \in C\right\},
$$

the following result holds.

Proposition 3.2. Let $C$ be a convex subset of $X$ and $x \in C$. Then $x \in$ qri $C$ if and only if $N_{C}(x)$ is a linear subspace of $X^{*}$.

Using the notion of qri $C$, in [17], the following separation theorem is proved.

Theorem 3.3. Let $C$ be a convex subset of $X$ and $x_{0} \in C \backslash$ qri $C$. Then, there exists $\xi \in X^{*}, \xi \neq \theta_{X^{*}}$, such that

$$
\langle\xi, x\rangle \leq\left\langle\xi, x_{0}\right\rangle, \quad \forall x \in C .
$$

Vice versa, let one suppose that there exist $\xi \neq \theta_{X^{*}}$ and a point $x_{0} \in X$ such that $\langle\xi, x\rangle \leq\left\langle\xi, x_{0}\right\rangle$, for all $x \in C$, and that $\mathrm{Cl}\left(\mathrm{T}_{\mathrm{C}}\left(\mathrm{x}_{0}\right)-\mathrm{T}_{\mathrm{C}}\left(\mathrm{x}_{0}\right)\right)=\mathrm{X}$. Then, $x_{0} \notin$ qri $C$.

Now, let us present the statement of the infinite dimensional duality theory.

Let $X$ be a real linear topological space and $S$ a nonempty convex subset of $X$; let $\left(Y,\|\cdot\|_{Y}\right)$ be a real normed space partially ordered by a convex cone $C$ and let $\left(Z,\|\cdot\|_{Z}\right)$ be a real normed space. Let $f: S \rightarrow \mathbb{R}$ and $g: S \rightarrow Y$ be two convex functions and let $h: S \rightarrow Z$ be an affine-linear function.

Let us consider the problem

$$
\min _{x \in \mathbb{K}} f(x),
$$

where $\mathbb{K}=\left\{x \in S: g(x) \in-C, h(x)=\theta_{Z}\right\}$, and the dual problem

$$
\max _{\substack{u \in C^{*} \\ v \in Z^{*}}} \inf _{x \in S}\{f(x)+\langle u, g(x)\rangle+\langle v, h(x)\rangle\}
$$

where $C^{*}=\left\{u \in Y^{*}:\langle u, y\rangle \geq 0 \forall y \in C\right\}$ is the dual cone of $C$.

We say that Assumption $S$ is fulfilled at a point $x_{0} \in \mathbb{K}$ if and only if it results in

$$
T_{\widetilde{M}}\left(0, \theta_{Y}, \theta_{Z}\right) \cap(]-\infty, 0\left[\times\left\{\theta_{Y}\right\} \times\left\{\theta_{Z}\right\}\right)=\emptyset,
$$

where $\widetilde{M}=\left\{\left(f(x)-f\left(x_{0}\right)+\alpha, g(x)+y, h(x)\right): x \in S \backslash \mathbb{K}, \alpha \geq 0, y \in C\right\}$.

Remark 3.4. If $\left(0, \theta_{Y}, \theta_{Z}\right) \notin \mathrm{Cl}(\widetilde{M})$, then Assumption $S$ holds, because $T_{\widetilde{M}}\left(0, \theta_{Y}, \theta_{Z}\right)=\emptyset$. 
Remark 3.5. If Assumption S holds, $T_{\widetilde{M}}\left(0, \theta_{Y}, \theta_{Z}\right) \neq \emptyset$, and $\left(l, \theta_{Y}, \theta_{Z}\right) \in T_{\widetilde{M}}\left(0, \theta_{Y}, \theta_{Z}\right)$, then $l \geq 0$.

Remark 3.6. If Assumption S holds, then $\left(0, \theta_{Y}, \theta_{Z}\right) \notin$ qri $\widetilde{M}$.

The following theorem holds (see [16]).

Theorem 3.7. Under the above assumptions, if problem (3.5) is solvable and Assumption $S$ is fulfilled at the extremal solution $x_{0} \in \mathbb{K}$, then also problem (3.6) is solvable, the extreme values of both problems are equal, and if $\left(x_{0}, \bar{u}, \bar{v}\right) \in \mathbb{K} \times C^{*} \times Z^{*}$ is the optimal point of problem (3.6), it results in:

$$
\left\langle\bar{u}, g\left(x_{0}\right)\right\rangle=0
$$

Using Theorem 3.7, we are able to show the usual relationship between a saddle point of the so-called Lagrange functional:

$$
\mathcal{L}(x, u, v)=f(x)+\langle u, g(x)\rangle+\langle v, h(x)\rangle, \quad \forall x \in S, \forall u \in C^{*}, \forall v \in Z^{*},
$$

and the solution of the constraint optimization problem (3.5) (see [16]).

Theorem 3.8. Let one assume that the assumptions of Theorem 3.7 are satisfied. Then, $x_{0} \in \mathbb{K}$ is a minimal solution to problem (3.5) if and only if there exist $\bar{u} \in C^{*}$ and $\bar{v} \in Z^{*}$ such that $\left(x_{0}, \bar{u}, \bar{v}\right)$ is a saddle point of the Lagrange functional (3.9), namely:

$$
\mathcal{L}\left(x_{0}, u, v\right) \leq \mathcal{L}\left(x_{0}, \bar{u}, \bar{v}\right) \leq \mathcal{L}(x, \bar{u}, \bar{v}), \quad \forall x \in S, \quad u \in C^{*}, v \in Z^{*},
$$

and, moreover, it results in

$$
\left\langle\bar{u}, g\left(x_{0}\right)\right\rangle=0
$$

\section{Proof of Existence of Lagrange Variables}

In this section, making use of the infinite dimensional Lagrange duality theory shown in Section 3, we will prove that equilibrium conditions (2.21)-(2.25) can be equivalently expressed by the evolutionary variational inequality (2.20). As a consequence, we determine under assumptions (i), (ii), (iii) on the profit function $v$, the equivalence with dynamic Cournot-Nash equilibrium conditions (2.18).

In order to prove Theorem 2.4, let us show some preliminary results. At first we recall Lemma 3.7 in [13] for the capacity constraints of the commodity shipments.

Lemma 4.1. Let $x^{*} \in \mathbb{K}$ be a solution to the variational inequality (2.20) and let one set

$$
\begin{aligned}
E_{i j}^{-} & =\left\{t \in[0, T]: x_{i j}^{*}(t)=\underline{x}_{i j}(t)\right\}, \quad \forall i=1, \ldots, m, \forall j=1, \ldots, n, \\
E_{i j}^{0} & =\left\{t \in[0, T]: \underline{x}_{i j}(t)<x_{i j}^{*}(t)<\bar{x}_{i j}(t)\right\}, \quad \forall i=1, \ldots, m, \forall j=1, \ldots, n, \\
E_{i j}^{+} & =\left\{t \in[0, T]: x_{i j}^{*}(t)=\bar{x}_{i j}(t)\right\}, \quad \forall i=1, \ldots, m, \forall j=1, \ldots, n .
\end{aligned}
$$


Then, one has

$$
\begin{array}{ll}
\frac{\partial v_{i}(t, \underline{x}(t))}{\partial x_{i j}} \leq 0, & \text { a.e. in } E_{i j^{\prime}}^{-} \\
\frac{\partial v_{i}\left(t, x^{*}(t)\right)}{\partial x_{i j}}=0, & \text { a.e. in } E_{i j^{\prime}}^{0} \\
\frac{\partial v_{i}(t, \bar{x}(t))}{\partial x_{i j}} \geq 0, & \text { a.e. in } E_{i j}^{+} .
\end{array}
$$

Now, we recall Lemma 4.1 in [1] that holds when production excesses occur.

Lemma 4.2. Let $x^{*} \in \mathbb{K}$ be a solution to the variational inequality (2.20). Setting

$$
\begin{aligned}
I_{i}^{0} & =\left\{t \in[0, T]: \sum_{j=1}^{n} x_{i j}^{*}(t)=p_{i}(t)\right\}, \quad i=1, \ldots, m, \\
r_{i}^{*}(t) & =\min \left\{-\frac{\partial v_{i}\left(t, x^{*}(t)\right)}{\partial x_{i j}}, j=1, \ldots, n\right\}, \quad \text { a.e. in } I_{i}^{0}, \quad i=1, \ldots, m, \\
X_{i}^{0} & =\left\{t \in I_{i}^{0}:-\frac{\partial v_{i}\left(t, x^{*}(t)\right)}{\partial x_{i j}}>\gamma_{i}^{*}(t)\right\}, \quad i=1, \ldots, m, \\
Y_{i}^{0} & =\left\{t \in I_{i}^{0}:-\frac{\partial v_{i}\left(t, x^{*}(t)\right)}{\partial x_{i j}}=r_{i}^{*}(t)\right\}, \quad i=1, \ldots, m,
\end{aligned}
$$

one has

$$
\begin{gathered}
\left(-\frac{\partial v_{i}\left(t, x^{*}(t)\right)}{\partial x_{i j}}-r_{i}^{*}(t)\right) x_{i j}^{*}(t)=0, \quad \text { a.e. in } I_{i}^{0}, \forall i=1, \ldots, m, \\
r_{i}^{*}(t) \leq 0, \quad \text { a.e. in } Y_{i}^{0}, \forall i=1, \ldots, m, \\
-\frac{\partial v_{i}\left(t, x^{*}(t)\right)}{\partial x_{i j}} \geq 0, \quad \text { a.e. in } X_{i}^{0}, \forall j=1, \ldots, n .
\end{gathered}
$$

With the same technique used for proving Lemma 4.2, we can obtain the following analogous result that holds when demand excesses occur.

Lemma 4.3. Let $x^{*} \in \mathbb{K}$ be a solution to the variational inequality (2.20). Setting

$$
\begin{aligned}
H_{j}^{0} & =\left\{t \in[0, T]: \sum_{i=1}^{m} x_{i j}^{*}(t)=q_{j}(t)\right\}, \quad j=1, \ldots, n, \\
\eta_{j}^{*}(t) & =\min \left\{-\frac{\partial v_{i}\left(t, x^{*}(t)\right)}{\partial x_{i j}}, i=1, \ldots, m\right\}, \quad \text { a.e. in } H_{j}^{0}, j=1, \ldots, n,
\end{aligned}
$$


Abstract and Applied Analysis

$$
\begin{aligned}
& V_{j}^{0}=\left\{t \in H_{j}^{0}:-\frac{\partial v_{i}\left(t, x^{*}(t)\right)}{\partial x_{i j}}>\eta_{j}^{*}(t)\right\}, \quad j=1, \ldots, n, \\
& W_{j}^{0}=\left\{t \in H_{j}^{0}:-\frac{\partial v_{i}\left(t, x^{*}(t)\right)}{\partial x_{i j}}=\eta_{j}^{*}(t)\right\}, \quad j=1, \ldots, n,
\end{aligned}
$$

one has

$$
\begin{gathered}
\left(-\frac{\partial v_{i}\left(t, x^{*}(t)\right)}{\partial x_{i j}}-\eta_{j}^{*}(t)\right) x_{i j}^{*}(t)=0, \text { a.e. in } H_{j}^{0}, j=1, \ldots, n, \\
\eta_{j}^{*}(t) \leq 0, \quad \text { a.e. in } W_{j}^{0}, \forall j=1, \ldots, n, \\
-\frac{\partial v_{i}\left(t, x^{*}(t)\right)}{\partial x_{i j}} \geq 0, \text { a.e. in } V_{j}^{0}, \forall i=1, \ldots, m .
\end{gathered}
$$

Now, we remember Lemma 4.2 in [1] that holds when production excesses occur.

Lemma 4.4. Let $x^{*} \in \mathbb{K}$ be a solution to the variational inequality (2.20). Setting

$$
I_{i}^{-}=\left\{t \in[0, T]: \sum_{j=1}^{n} x_{i j}^{*}(t)-p_{i}(t)<0\right\}, \quad i=1, \ldots, m,
$$

one has

$$
-\frac{\partial v_{i}\left(t, x^{*}(t)\right)}{\partial x_{i j}}=0, \quad \text { a.e. in } I_{i}^{-}, \forall i=1, \ldots, m
$$

Finally, by proceeding as in Lemma 4.4 we can prove the following analogous result that holds when demand excesses occur.

Lemma 4.5. Let $x^{*} \in \mathbb{K}$ be a solution to the variational inequality (2.20). Setting

$$
H_{j}^{-}=\left\{t \in[0, T]: \sum_{i=1}^{m} x_{i j}^{*}(t)-q_{j}(t)<0\right\}, \quad j=1, \ldots, n,
$$

one has

$$
-\frac{\partial v_{i}\left(t, x^{*}(t)\right)}{\partial x_{i j}}=0, \quad \text { a.e. in } H_{j}^{-}, \forall j=1, \ldots, n
$$

Now we are able to prove Theorem 2.4. 
Proof of Theorem 2.4. Let us assume that $x^{*} \in \mathbb{K}$ is an equilibrium solution according to Definition 2.3. Then, taking into account that $\lambda_{i j}^{*}(t)\left(\underline{x}_{i j}(t)-x_{i j}^{*}(t)\right)=0$ and $\rho_{i j}^{*}(t)\left(x_{i j}^{*}(t)-\bar{x}_{i j}(t)\right)=$ 0 , a.e. in $[0, T]$, we have for every $x \in \mathbb{K}$, a.e. in $[0, T]$,

$$
\begin{aligned}
-\frac{\partial v_{i}\left(t, x^{*}(t)\right)}{\partial x_{i j}}\left(x_{i j}(t)-x_{i j}^{*}(t)\right)= & -\rho_{i j}^{*}(t)\left(x_{i j}(t)-x_{i j}^{*}(t)\right)-\mu_{i}^{*}(t)\left(x_{i j}(t)-x_{i j}^{*}(t)\right) \\
& -v_{j}^{*}(t)\left(x_{i j}(t)-x_{i j}^{*}(t)\right)+\lambda_{i j}^{*}(t)\left(x_{i j}(t)-x_{i j}^{*}(t)\right) \\
= & -\rho_{i j}^{*}(t)\left(x_{i j}(t)-\bar{x}_{i j}(t)\right)-\mu_{i}^{*}(t)\left(x_{i j}(t)-x_{i j}^{*}(t)\right) \\
& -v_{j}^{*}(t)\left(x_{i j}(t)-x_{i j}^{*}(t)\right)+\lambda_{i j}^{*}(t)\left(x_{i j}(t)-\underline{x}_{i j}(t)\right) \\
\geq & -\mu_{i}^{*}(t)\left(x_{i j}(t)-x_{i j}^{*}(t)\right)-v_{j}^{*}(t)\left(x_{i j}(t)-x_{i j}^{*}(t)\right),
\end{aligned}
$$

and, as a consequence, by summing over $i=1, \ldots, m$ and $j=1, \ldots, n$, integrating on $[0, T]$ and using the conditions (2.24) and (2.25), it results, for each $x \in \mathbb{K}$

$$
\begin{aligned}
\int_{0}^{T} \sum_{i=1}^{m} \sum_{j=1}^{n}-\frac{\partial v_{i}\left(t, x^{*}(t)\right)}{\partial x_{i j}}\left(x_{i j}(t)-x_{i j}^{*}(t)\right) d t \\
\geq-\sum_{i=1}^{m} \int_{0}^{T} \mu_{i}^{*}(t)\left(\sum_{j=1}^{n} x_{i j}(t)-\sum_{j=1}^{n} x_{i j}^{*}(t)+p_{i}(t)-p_{i}(t)\right) d t \\
\quad-\sum_{j=1}^{n} \int_{0}^{T} v_{j}^{*}(t)\left(\sum_{i=1}^{m} x_{i j}(t)-\sum_{i=1}^{m} x_{i j}^{*}(t)+q_{j}(t)-q_{j}(t)\right) d t \\
=-\sum_{i=1}^{m} \int_{0}^{T} \mu_{i}^{*}(t)\left(\sum_{j=1}^{n} x_{i j}(t)-p_{i}(t)\right) d t \\
\quad-\sum_{j=1}^{n} \int_{0}^{T} v_{j}^{*}(t)\left(\sum_{i=1}^{m} x_{i j}(t)-q_{j}(t)\right) d t \geq 0 .
\end{aligned}
$$

Hence, we obtain (2.20).

Vice versa, let $x^{*} \in \mathbb{K}$ be a solution to (2.20) and let us apply the infinite dimensional duality theory. First of all, let us prove that the Assumption $\mathrm{S}$ is fulfilled. 
Let us set, for $x \in L^{2}\left([0, T], \mathbb{R}^{m n}\right)$,

$$
\begin{aligned}
& \Psi(x)=\int_{0}^{T} \sum_{i=1}^{m} \sum_{j=1}^{n}-\frac{\partial v_{i}\left(t, x^{*}(t)\right)}{\partial x_{i j}}\left(x_{i j}(t)-x_{i j}^{*}(t)\right) d t, \\
& \widetilde{M}=\left\{\left(\Psi(x)+\alpha,-x+\underline{x}+y, x-\bar{x}+u, \sum_{j=1}^{n} x_{i j}-p_{i}+v_{i}, \sum_{i=1}^{m} x_{i j}-q_{j}+z_{j}\right):\right. \\
& i=1, \ldots, m, j=1, \ldots, n, \alpha \geq 0, x \in L^{2}\left([0, T], \mathbb{R}_{+}^{m n}\right) \backslash \mathbb{K}, \\
&\left.y, u \in L^{2}\left([0, T], \mathbb{R}_{+}^{m n}\right), v \in L^{2}\left([0, T], \mathbb{R}_{+}^{m}\right), z \in L^{2}\left([0, T], \mathbb{R}_{+}^{n}\right)\right\},
\end{aligned}
$$

we must show that if $\left(l, \theta_{L^{2}\left([0, T], \mathbb{R}_{+}^{m n}\right)}, \theta_{L^{2}\left([0, T], \mathbb{R}_{+}^{m n}\right)}, \theta_{L^{2}\left([0, T], \mathbb{R}_{+}^{m}\right)}, \theta_{L^{2}\left([0, T], \mathbb{R}_{+}^{n}\right)}\right)$ belongs to $T_{\widetilde{M}^{(}}\left(0, \theta_{L^{2}\left([0, T], \mathbb{R}_{+}^{m n}\right)}, \theta_{L^{2}\left([0, T], \mathbb{R}_{+}^{m n}\right)}, \theta_{L^{2}\left([0, T], \mathbb{R}_{+}^{m}\right)}, \theta_{L^{2}\left([0, T], \mathbb{R}_{+}^{n}\right)}\right)$, namely:

$$
\begin{aligned}
& \lim _{n \rightarrow+\infty} \lambda_{n}\left(\Psi\left(x^{n}\right)+\alpha_{n}\right)=l, \\
& \lim _{n \rightarrow+\infty} \lambda_{n}\left(-x^{n}+\underline{x}+y^{n}\right)=\theta_{L^{2}\left([0, T], \mathbb{R}_{+}^{m n}\right),} \\
& \lim _{n \rightarrow+\infty} \lambda_{n}\left(x^{n}-\bar{x}+u^{n}\right)=\theta_{L^{2}\left([0, T], \mathbb{R}_{+}^{m n}\right),} \\
& \lim _{n \rightarrow+\infty} \lambda_{n}\left(\sum_{j=1}^{n} x_{i j}^{n}-p_{i}+v_{i}^{n}\right)=\theta_{L^{2}\left([0, T], \mathbb{R}_{+}\right),} \quad \forall i=1, \ldots, m, \\
& \lim _{n \rightarrow+\infty} \lambda_{n}\left(\sum_{i=1}^{m} x_{i j}^{n}-q_{j}+z_{j}^{n}\right)=\theta_{L^{2}\left([0, T], \mathbb{R}_{+}\right)}, \quad \forall j=1, \ldots, n,
\end{aligned}
$$

with $\lambda_{n} \geq 0, x^{n} \in L^{2}\left([0, T], \mathbb{R}_{+}^{m n}\right) \backslash \mathbb{K}, \alpha_{n} \geq 0, y^{n}, u^{n} \in L^{2}\left([0, T], \mathbb{R}_{+}^{m n}\right), v^{n} \in L^{2}\left([0, T], \mathbb{R}_{+}^{m}\right)$, $z^{n} \in L^{2}\left([0, T], \mathbb{R}_{+}^{n}\right)$, for all $n \in \mathbb{N}$, and

$$
\begin{aligned}
& \lim _{n \rightarrow+\infty}\left(\Psi\left(x^{n}\right)+\alpha_{n}\right)=0, \\
& \lim _{n \rightarrow+\infty}\left(-x^{n}+\underline{x}+y^{n}\right)=\theta_{L^{2}\left([0, T], \mathbb{R}_{+}^{m n}\right),} \\
& \lim _{n \rightarrow+\infty}\left(x^{n}+\bar{x}+u^{n}\right)=\theta_{L^{2}\left([0, T], \mathbb{R}_{+}^{m n}\right),} \\
& \lim _{n \rightarrow+\infty}\left(\sum_{j=1}^{n} x_{i j}^{n}-p_{i}+v_{i}^{n}\right)=\theta_{L^{2}\left([0, T], \mathbb{R}_{+}\right),} \quad \forall i=1, \ldots, m, \\
& \lim _{n \rightarrow+\infty}\left(\sum_{i=1}^{m} x_{i j}^{n}-q_{j}+z_{j}^{n}\right)=\theta_{L^{2}\left([0, T], \mathbb{R}_{+}\right)}, \quad \forall j=1, \ldots, n,
\end{aligned}
$$

then $l$ is nonnegative. 
Let us set

$$
\begin{aligned}
& E_{i j}^{-}=\left\{t \in[0, T]: x_{i j}^{*}(t)=\underline{x}_{i j}(t)\right\}, \quad i=1, \ldots, m, j=1, \ldots, n, \\
& E_{i j}^{0}=\left\{t \in[0, T]: \underline{x}_{i j}(t)<x_{i j}^{*}(t)<\bar{x}_{i j}(t)\right\}, \quad i=1, \ldots, m, j=1, \ldots, n, \\
& E_{i j}^{+}=\left\{t \in[0, T]: x_{i j}^{*}(t)=\bar{x}_{i j}(t)\right\}, \quad i=1, \ldots, m, j=1, \ldots, n, \\
& I_{i}^{0}=\left\{t \in[0, T]: \sum_{j=1}^{n} x_{i j}^{*}(t)=p_{i}(t)\right\}, \quad i=1, \ldots, m, \\
& H_{j}^{0}=\left\{t \in[0, T]: \sum_{i=1}^{m} x_{i j}^{*}(t)=q_{j}(t)\right\}, \quad j=1, \ldots, n, \\
& I_{i}^{-}=\left\{t \in[0, T]: \sum_{j=1}^{n} x_{i j}^{*}(t)-p_{i}(t)<0\right\}, \quad i=1, \ldots, m, \\
& H_{j}^{-}=\left\{t \in[0, T]: \sum_{i=1}^{m} x_{i j}^{*}(t)-q_{j}(t)<0\right\}, \quad j=1, \ldots, n, \\
& r_{i}^{*}(t)=\min \left\{-\frac{\partial v_{i}\left(t, x^{*}(t)\right)}{\partial x_{i j}}, j=1, \ldots, n\right\}, \quad t \in I_{i}^{0}, i=1, \ldots, m \text {, } \\
& \eta_{j}^{*}(t)=\min \left\{-\frac{\partial v_{i}\left(t, x^{*}(t)\right)}{\partial x_{i j}}, i=1, \ldots, m\right\}, \quad t \in H_{j}^{0}, j=1, \ldots, n, \\
& X_{i}^{0}=\left\{t \in I_{i}^{0}:-\frac{\partial v_{i}\left(t, x^{*}(t)\right)}{\partial x_{i j}}>\gamma_{i}^{*}(t)\right\}, \quad i=1, \ldots, m, \\
& Y_{i}^{0}=\left\{t \in I_{i}^{0}:-\frac{\partial v_{i}\left(t, x^{*}(t)\right)}{\partial x_{i j}}=\gamma_{i}^{*}(t)\right\}, \quad i=1, \ldots, m, \\
& V_{j}^{0}=\left\{t \in H_{j}^{0}:-\frac{\partial v_{i}\left(t, x^{*}(t)\right)}{\partial x_{i j}}>\eta_{j}^{*}(t)\right\}, \quad j=1, \ldots, n, \\
& W_{j}^{0}=\left\{t \in H_{j}^{0}:-\frac{\partial v_{i}\left(t, x^{*}(t)\right)}{\partial x_{i j}}=\eta_{j}^{*}(t)\right\}, \quad j=1, \ldots, n .
\end{aligned}
$$

Before starting with the proof let us observe the following:

$$
[0, T]=I_{i}^{0} \cup\left(H_{j}^{0} \backslash I_{i}^{0}\right) \cup\left(I_{i}^{-} \cap H_{j}^{-}\right), \quad \forall i=1, \ldots, m, \forall j=1, \ldots, n,
$$

and also

$$
[0, T]=E_{i j}^{-} \cup E_{i j}^{0} \cup E_{i j}^{+}, \quad \forall i=1, \ldots, m, \forall j=1, \ldots, n .
$$


Moreover,

$$
\begin{aligned}
E_{i j}^{-}= & E_{i j}^{-} \cap\left[I_{i}^{0} \cup\left(H_{j}^{0} \backslash I_{i}^{0}\right) \cup\left(I_{i}^{-} \cap H_{j}^{-}\right)\right] \\
= & \left(E_{i j}^{-} \cap I_{i}^{0}\right) \cup\left[E_{i j}^{-} \cap\left(H_{j}^{0} \backslash I_{i}^{0}\right)\right] \cup\left[E_{i j}^{-} \cap\left(I_{i}^{-} \cap H_{j}^{-}\right)\right] \\
= & \left(E_{i j}^{-} \cap X_{i}^{0}\right) \cup\left(E_{i j}^{-} \cap Y_{i}^{0}\right) \cup\left[E_{i j}^{-} \cap\left(V_{j}^{0} \backslash I_{i}^{0}\right)\right] \\
& \cup\left[E_{i j}^{-} \cap\left(W_{j}^{0} \backslash I_{i}^{0}\right)\right] \cup\left[E_{i j}^{-} \cap\left(I_{i}^{-} \cap H_{j}^{-}\right)\right], \quad \forall i=1, \ldots, m, \quad \forall j=1, \ldots, n,
\end{aligned}
$$

and, analogously,

$$
\begin{aligned}
E_{i j}^{0}= & \left(E_{i j}^{0} \cap X_{i}^{0}\right) \cup\left(E_{i j}^{0} \cap Y_{i}^{0}\right) \cup\left[E_{i j}^{0} \cap\left(V_{j}^{0} \backslash I_{i}^{0}\right)\right] \cup\left[E_{i j}^{0} \cap\left(W_{j}^{0} \backslash I_{i}^{0}\right)\right] \\
& \cup\left[E_{i j}^{0} \cap\left(I_{i}^{-} \cap H_{j}^{-}\right)\right], \quad \forall i=1, \ldots, m, \forall j=1, \ldots, n, \\
E_{i j}^{+}= & \left(E_{i j}^{+} \cap X_{i}^{0}\right) \cup\left(E_{i j}^{+} \cap Y_{i}^{0}\right) \cup\left[E_{i j}^{+} \cap\left(V_{j}^{0} \backslash I_{i}^{0}\right)\right] \\
& \cup\left[E_{i j}^{+} \cap\left(W_{j}^{0} \backslash I_{i}^{0}\right)\right] \cup\left[E_{i j}^{+} \cap\left(I_{i}^{-} \cap H_{j}^{-}\right)\right], \quad \forall i=1, \ldots, m, \forall j=1, \ldots, n .
\end{aligned}
$$

Now we observe that, for Lemmas 4.1, 4.2, 4.3, 4.4, and 4.5, we get

$$
\begin{aligned}
& -\frac{\partial v_{i}\left(t, x^{*}(t)\right)}{\partial x_{i j}}=0, \text { a.e. in } E_{i j}^{0}, \quad \forall i=1, \ldots, m, \forall j=1, \ldots, n, \\
& -\frac{\partial v_{i}(t, \underline{x}(t))}{\partial x_{i j}} \geq 0, \text { a.e. in } E_{i j}^{-} \cap X_{i}^{0}, \quad \forall i=1, \ldots, m, \forall j=1, \ldots, n, \\
& -\frac{\partial v_{i}(t, \underline{x}(t))}{\partial x_{i j}}=\gamma_{i}^{*}(t)=0, \text { a.e. in } E_{i j}^{-} \cap Y_{i}^{0}, \quad \forall i=1, \ldots, m, \forall j=1, \ldots, n, \\
& -\frac{\partial v_{i}(t, \underline{x}(t))}{\partial x_{i j}} \geq 0, \text { a.e. in } E_{i j}^{-} \cap\left(V_{j}^{0} \backslash I_{i}^{0}\right), \quad \forall i=1, \ldots, m, \forall j=1, \ldots, n, \\
& -\frac{\partial v_{i}(t, \underline{x}(t))}{\partial x_{i j}}=\eta_{j}^{*}(t)=0, \text { a.e. in } E_{i j}^{-} \cap\left(W_{j}^{0} \backslash I_{i}^{0}\right), \quad \forall i=1, \ldots, m, \forall j=1, \ldots, n, \\
& -\frac{\partial v_{i}(t, \underline{x}(t))}{\partial x_{i j}}=0, \text { a.e. in } E_{i j}^{-} \cap\left(I_{i}^{-} \cap H_{j}^{-}\right), \quad \forall i=1, \ldots, m, \forall j=1, \ldots, n, \\
& -\frac{\partial v_{i}(t, \bar{x}(t))}{\partial x_{i j}}=0, \text { a.e. in } E_{i j}^{+} \cap X_{i}^{0}, \quad \forall i=1, \ldots, m, \forall j=1, \ldots, n,
\end{aligned}
$$




$$
\begin{aligned}
& -\frac{\partial v_{i}(t, \bar{x}(t))}{\partial x_{i j}}=\gamma_{i}^{*}(t) \leq 0, \text { a.e. in } E_{i j}^{+} \cap Y_{i}^{0}, \quad \forall i=1, \ldots, m, \forall j=1, \ldots, n, \\
& -\frac{\partial v_{i}(t, \bar{x}(t))}{\partial x_{i j}}=0, \text { a.e. in } E_{i j}^{+} \cap\left(V_{j}^{0} \backslash I_{i}^{0}\right), \quad \forall i=1, \ldots, m, \forall j=1, \ldots, n, \\
& -\frac{\partial v_{i}(t, \bar{x}(t))}{\partial x_{i j}}=\eta_{j}^{*}(t) \leq 0, \text { a.e. in } E_{i j}^{+} \cap\left(W_{j}^{0} \backslash I_{i}^{0}\right), \quad \forall i=1, \ldots, m, \forall j=1, \ldots, n, \\
& -\frac{\partial v_{i}(t, \bar{x}(t))}{\partial x_{i j}}=0, \text { a.e. in } E_{i j}^{+} \cap\left(I_{i}^{-} \cap H_{j}^{-}\right), \quad \forall i=1, \ldots, m, \forall j=1, \ldots, n .
\end{aligned}
$$

As a consequence, we have

$$
\begin{array}{r}
l=\lim _{n \rightarrow+\infty} \lambda_{n}\left(\Psi\left(x^{n}\right)+\alpha_{n}\right) \\
=\lim _{n \rightarrow+\infty} \lambda_{n}\left(\int_{0}^{T} \sum_{i=1}^{m} \sum_{j=1}^{n}-\frac{\partial v_{i}\left(t, x^{*}(t)\right)}{\partial x_{i j}}\left(x_{i j}^{n}(t)-x_{i j}^{*}(t)\right) d t+\alpha_{n}\right) \\
\geq \lim _{n \rightarrow+\infty} \lambda_{n}\left(\int_{E_{i j}^{-}} \sum_{i=1}^{m} \sum_{j=1}^{n}-\frac{\partial v_{i}\left(t, x^{*}(t)\right)}{\partial x_{i j}}\left(x_{i j}^{n}(t)-\underline{x}_{i j}(t)\right) d t\right. \\
\quad+\int_{E_{i j}^{0}} \sum_{i=1}^{m} \sum_{j=1}^{n}-\frac{\partial v_{i}\left(t, x^{*}(t)\right)}{\partial x_{i j}}\left(x_{i j}^{n}(t)-x_{i j}^{*}(t)\right) d t \\
\left.\quad+\int_{E_{i j}^{*}} \sum_{i=1}^{m} \sum_{j=1}^{n}-\frac{\partial v_{i}\left(t, x^{*}(t)\right)}{\partial x_{i j}}\left(x_{i j}^{n}(t)-\bar{x}_{i j}(t)\right) d t\right) .
\end{array}
$$

We note that

$$
\lim _{n \rightarrow+\infty} \lambda_{n} \int_{E_{i j}^{0}} \sum_{i=1}^{m} \sum_{j=1}^{n}-\frac{\partial v_{i}\left(t, x^{*}(t)\right)}{\partial x_{i j}}\left(x_{i j}^{n}(t)-x_{i j}^{*}(t)\right) d t=0
$$

being $-\partial v_{i}\left(t, x^{*}(t)\right) / \partial x_{i j}=0$, a.e. in $E_{i j}^{0}$, for all $i=1, \ldots, m$, for all $j=1, \ldots, n$. We will prove that

$$
\begin{aligned}
& \lim _{n \rightarrow+\infty} \lambda_{n} \int_{E_{i j}^{-}} \sum_{i=1}^{m} \sum_{j=1}^{n}-\frac{\partial v_{i}(t, \underline{x}(t))}{\partial x_{i j}}\left(x_{i j}^{n}(t)-\underline{x}_{i j}(t)\right) d t \geq 0, \\
& \lim _{n \rightarrow+\infty} \lambda_{n} \int_{E_{i j}^{+}} \sum_{i=1}^{m} \sum_{j=1}^{n}-\frac{\partial v_{i}(t, \bar{x}(t))}{\partial x_{i j}}\left(x_{i j}^{n}(t)-\bar{x}_{i j}(t)\right) d t \geq 0 .
\end{aligned}
$$


In fact, it results in

$$
\begin{aligned}
\lambda_{n} \int_{E_{i j}^{-}} \sum_{i=1}^{m} \sum_{j=1}^{n}-\frac{\partial v_{i}(t, \underline{x}(t))}{\partial x_{i j}}\left(x_{i j}^{n}(t)-\underline{x}_{i j}(t)\right) d t \\
=\lambda_{n} \int_{E_{i j}^{-} \cap X_{i}^{0}} \sum_{i=1}^{m} \sum_{j=1}^{n}-\frac{\partial v_{i}(t, \underline{x}(t))}{\partial x_{i j}}\left(x_{i j}^{n}(t)-\underline{x}_{i j}(t)\right) d t \\
\quad+\lambda_{n} \int_{E_{i j}^{-} \cap Y_{i}^{0}} \sum_{i=1}^{m} \sum_{j=1}^{n}-\frac{\partial v_{i}(t, \underline{x}(t))}{\partial x_{i j}}\left(x_{i j}^{n}(t)-\underline{x}_{i j}(t)\right) d t \\
+\lambda_{n} \int_{E_{i j}^{-} \cap\left(V_{j}^{0} \backslash I_{i}^{0}\right)} \sum_{i=1}^{m} \sum_{j=1}^{n}-\frac{\partial v_{i}(t, \underline{x}(t))}{\partial x_{i j}}\left(x_{i j}^{n}(t)-\underline{x}_{i j}(t)\right) d t \\
\quad+\lambda_{n} \int_{E_{i j}^{-} \cap\left(W_{j}^{0} \backslash I_{i}^{0}\right)} \sum_{i=1}^{m} \sum_{j=1}^{n}-\frac{\partial v_{i}(t, \underline{x}(t))}{\partial x_{i j}}\left(x_{i j}^{n}(t)-\underline{x}_{i j}(t)\right) d t \\
\quad+\lambda_{n} \int_{E_{i j}^{-} \cap I_{i}^{-} \cap H_{j}^{-}} \sum_{i=1}^{m} \sum_{j=1}^{n}-\frac{\partial v_{i}(t, \underline{x}(t))}{\partial x_{i j}^{n}}\left(x_{i j}^{n}(t)-\underline{x}_{i j}(t)\right) d t \\
=\lambda_{n} \int_{E_{i j}^{-} \cap X_{i}^{0}} \sum_{i=1}^{m} \sum_{j=1}^{n}-\frac{\partial v_{i}(t, \underline{x}(t))}{\partial x_{i j}} x_{i j}^{n}(t) d t \\
\quad+\lambda_{n} \int_{E_{i j}^{-} \cap\left(V_{j}^{0} \backslash I_{i}^{0}\right)} \sum_{i=1}^{m} \sum_{j=1}^{n}-\frac{\partial v_{i}(t, \underline{x}(t))}{\partial x_{i j}} x_{i j}^{n}(t) d t .
\end{aligned}
$$

By virtue of the previous remarks, conditions (4.4) and (4.6), Lemmas 4.1, 4.2, 4.3, 4.4, and 4.5 , for the conditions of belonging to the tangent cone, we get the first inequality of (4.25) and, with analogous considerations, we get the second inequality of (4.25).

Therefore, thanks to (4.25) and (4.24), we have that

$$
\begin{aligned}
l & =\lim _{n \rightarrow+\infty} \lambda_{n}\left(\Psi\left(x^{n}\right)+\alpha_{n}\right) \\
& =\lim _{n \rightarrow+\infty} \lambda_{n}\left(\int_{0}^{T} \sum_{i=1}^{m} \sum_{j=1}^{n}-\frac{\partial v_{i}\left(t, x^{*}(t)\right)}{\partial x_{i j}}\left(x_{i j}^{n}(t)-x_{i j}^{*}(t)\right) d t+\alpha_{n}\right)
\end{aligned}
$$

is nonnegative. 
Taking into account Theorems 3.7 and 3.8, if we consider the Lagrange function,

$$
\begin{aligned}
\mathcal{L}(x, \lambda, \rho, \mu, v)= & \Psi(x)-\sum_{i=1}^{m} \sum_{j=1}^{n} \int_{0}^{T} \lambda_{i j}(t)\left(\underline{x}_{i j}(t)-x_{i j}(t)\right) d t \\
& +\sum_{i=1}^{m} \sum_{j=1}^{n} \int_{0}^{T} \rho_{i j}(t)\left(x_{i j}(t)-\bar{x}_{i j}(t)\right) d t \\
& +\sum_{i=1}^{m} \int_{0}^{T} \mu_{i}(t)\left(\sum_{j=1}^{n} x_{i j}(t)-p_{i}(t)\right) d t \\
& +\sum_{j=1}^{n} \int_{0}^{T} v_{j}(t)\left(\sum_{i=1}^{m} x_{i j}(t)-q_{j}(t)\right) d t
\end{aligned}
$$

we have that there exist $\lambda^{*}, \rho^{*} \in L^{2}\left([0, T], \mathbb{R}_{+}^{m n}\right), \mu^{*} \in L^{2}\left([0, T], \mathbb{R}_{+}^{m}\right), v^{*} \in L^{2}\left([0, T], \mathbb{R}_{+}^{n}\right)$, such that

$$
\mathcal{L}\left(x^{*}, \lambda, \rho, \mu, v\right) \leq \mathcal{L}\left(x^{*}, \lambda^{*}, \rho^{*}, \mu^{*}, v^{*}\right) \leq \mathcal{L}\left(x, \lambda^{*}, \rho^{*}, \mu^{*}, v^{*}\right),
$$

for all $x \in L^{2}\left([0, T], \mathbb{R}_{+}^{m n}\right), \lambda, \rho \in L^{2}\left([0, T], \mathbb{R}_{+}^{m n}\right), \mu \in L^{2}\left([0, T], \mathbb{R}_{+}^{m}\right), v \in L^{2}\left([0, T], \mathbb{R}_{+}^{n}\right)$, and, moreover,

$$
\begin{aligned}
\lambda_{i j}^{*}(t)\left(\underline{x}_{i j}(t)-x_{i j}^{*}(t)\right) & =0, \quad \forall i=1, \ldots, m, \forall j=1, \ldots, n, \\
\rho_{i j}^{*}(t)\left(x_{i j}^{*}(t)-\bar{x}_{i j}(t)\right) & =0, \quad \forall i=1, \ldots, m, \forall j=1, \ldots, n, \\
\mu_{i}^{*}(t)\left(\sum_{j=1}^{n} x_{i j}^{*}(t)-p_{i}(t)\right) & =0, \quad \forall i=1, \ldots, m, \\
v_{j}^{*}(t)\left(\sum_{i=1}^{m} x_{i j}^{*}(t)-q_{j}(t)\right) & =0, \quad \forall j=1, \ldots, n .
\end{aligned}
$$


Then, for conditions (4.30), $\mathcal{L}\left(x^{*}, \lambda^{*}, \rho^{*}, \mu^{*}, \nu^{*}\right)=0$, and by virtue of the right-hand side of (4.29) and the equalities (4.30), we get

$$
\begin{aligned}
\mathcal{L}\left(x, \lambda^{*}, \rho^{*}, \mu^{*}, v^{*}\right)= & \sum_{i=1}^{m} \sum_{j=1}^{n} \int_{0}^{T}-\frac{\partial v_{i}\left(t, x^{*}(t)\right)}{\partial x_{i j}}\left(x_{i j}(t)-x_{i j}^{*}(t)\right) d t \\
& -\sum_{i=1}^{m} \sum_{j=1}^{n} \int_{0}^{T} \lambda_{i j}^{*}(t)\left(x_{i j}(t)-x_{i j}^{*}(t)\right) d t \\
& +\sum_{i=1}^{m} \sum_{j=1}^{n} \int_{0}^{T} \rho_{i j}^{*}(t)\left(x_{i j}(t)-x_{i j}^{*}(t)\right) d t \\
& +\sum_{i=1}^{m} \sum_{j=1}^{n} \int_{0}^{T} \mu_{i}^{*}(t)\left(x_{i j}(t)-x_{i j}^{*}(t)\right) d t \\
& +\sum_{j=1}^{n} \sum_{i=1}^{m} \int_{0}^{T} v_{j}^{*}(t)\left(x_{i j}(t)-x_{i j}^{*}(t)\right) d t \\
\geq & \mathcal{L}\left(x^{*}, \lambda^{*}, \rho^{*}, \mu^{*}, v^{*}\right)=0, \quad \forall x \in L^{2}\left([0, T], \mathbb{R}_{+}^{m n}\right) .
\end{aligned}
$$

Then, $\mathcal{L}\left(x, \lambda^{*}, \rho^{*}, \mu^{*}, \nu^{*}\right)$ has a minimal point in $x^{*}$.

Let us assume that $x_{i j}^{1}=x_{i j}^{*}+\epsilon_{i j}$ and $x_{i j}^{2}=x_{i j}^{*}-\epsilon_{i j}$, for all $\epsilon \in L^{2}\left([0, T], \mathbb{R}_{+}^{m n}\right)$. Let us note that

$$
\begin{gathered}
\mathcal{L}\left(x^{1}, \lambda^{*}, \rho^{*}, \mu^{*}, v^{*}\right) \\
=\sum_{i=1}^{m} \sum_{j=1}^{n} \int_{0}^{T}\left(-\frac{\partial v_{i}\left(t, x^{*}(t)\right)}{\partial x_{i j}}-\lambda_{i j}^{*}(t)+\rho_{i j}^{*}(t)+\mu_{i}^{*}(t)+v_{i}^{*}(t)\right) \epsilon_{i j}(t) d t \geq 0, \\
\forall \epsilon \in L^{2}\left([0, T], \mathbb{R}_{+}^{m n}\right), \\
\mathcal{L}\left(x^{2}, \lambda^{*}, \rho^{*}, \mu^{*}, v^{*}\right) \\
=-\left\{\sum_{i=1}^{m} \sum_{j=1}^{n} \int_{0}^{T}\left(-\frac{\partial v_{i}\left(t, x^{*}(t)\right)}{\partial x_{i j}}-\lambda_{i j}^{*}(t)+\rho_{i j}^{*}(t)+\mu_{i}^{*}(t)+v_{i}^{*}(t)\right) \epsilon_{i j}(t) d t\right\} \geq 0, \\
\forall \in \in L^{2}\left([0, T], \mathbb{R}_{+}^{m n}\right) .
\end{gathered}
$$

As a consequence, we have

$$
\begin{array}{r}
\sum_{\mathrm{I}=1}^{m} \sum_{j=1}^{n} \int_{0}^{T}\left(-\frac{\partial v_{i}\left(t, x^{*}(t)\right)}{\partial x_{i j}}-\lambda_{i j}^{*}(t)+\rho_{i j}^{*}(t)+\mu_{i}^{*}(t)+v_{i}^{*}(t)\right) \epsilon_{i j}(t) d t=0, \\
\forall \epsilon \in L^{2}\left([0, T], \mathbb{R}_{+}^{m n}\right),
\end{array}
$$


and, hence, we obtain the equilibrium conditions according to Definition 2.3, namely:

$$
\begin{gathered}
-\frac{\partial v_{i}\left(t, x^{*}(t)\right)}{\partial x_{i j}}+\rho_{i j}^{*}(t)+\mu_{i}^{*}(t)+v_{j}^{*}(t)=\lambda_{i j}^{*}(t), \\
\lambda_{i j}^{*}(t)\left(\underline{x}_{i j}(t)-x_{i j}^{*}(t)\right)=0, \quad \lambda_{i j}^{*}(t) \geq 0, \\
\rho_{i j}^{*}(t)\left(x_{i j}^{*}(t)-\bar{x}_{i j}(t)\right)=0, \quad \rho_{i j}^{*}(t) \geq 0, \\
\mu_{i}^{*}(t)\left(\sum_{j=1}^{n} x_{i j}^{*}(t)-p_{i}(t)\right)=0, \quad \mu_{i}^{*}(t) \geq 0, \\
v_{j}^{*}(t)\left(\sum_{i=1}^{m} x_{i j}^{*}(t)-q_{j}(t)\right)=0, \quad v_{j}^{*}(t) \geq 0,
\end{gathered}
$$

for all $i=1, \ldots, m, j=1, \ldots, n$, a.e. in $[0, T]$.

\section{Existence Results}

This section is devoted to show some results for the existence of solutions to the dynamic oligopolistic market equilibrium problem in presence of excesses.

Let us recall some definitions (see [24]). Let $X$ be a reflexive Banach space, let $\mathbb{K}$ be a subset of $X$, and let $X^{*}$ be the dual space of $X$.

Definition 5.1. A mapping $A: \mathbb{K} \rightarrow X^{*}$ is strongly monotone on $\mathbb{K}$ if and only if for all $u$, $v \in \mathbb{K}$, there exists $v>0$ such that $\langle A u-A v, u-v\rangle \geq v\|u-v\|_{\mathbb{K}}^{2}$.

Definition 5.2. A mapping $A: \mathbb{K} \rightarrow X^{*}$ is pseudomonotone in the sense of Karamardian (K-pseudomonotone) if and only if for all $u, v \in \mathbb{K}$,

$$
\langle A v, u-v\rangle \geq 0 \Longrightarrow\langle A u, u-v\rangle \geq 0
$$

Definition 5.3. A mapping $A: \mathbb{K} \rightarrow X^{*}$ is strictly pseudomonotone if and only if for all $u$, $v \in \mathbb{K}, u \neq v$,

$$
\langle A v, u-v\rangle \geq 0 \Longrightarrow\langle A u, u-v\rangle>0 .
$$

Definition 5.4. A mapping $A: \mathbb{K} \rightarrow X^{*}$ is pseudomonotone in the sense of Brezis (Bpseudomonotone) if and only if:

(1) for each sequence $\left\{u_{n}\right\}$ weakly converging to $u$ (in short $u_{n} \rightarrow u$ ) in $\mathbb{K}$ and such that $\lim \sup _{n}\left\langle A u_{n}, u_{n}-v\right\rangle \leq 0$ it results in

$$
\liminf \operatorname{in}_{n}\left\langle A u_{n}, u_{n}-v\right\rangle \geq\langle A u, u-v\rangle, \quad \forall v \in \mathbb{K}
$$


(2) for each $v \in \mathbb{K}$ the function $u \mapsto\langle A u, u-v\rangle$ is lower bounded on the bounded subset of $\mathbb{K}$.

Let, now, $\mathbb{K}$ be a convex subset of $X$.

Definition 5.5. A mapping $A: \mathbb{K} \rightarrow X^{*}$ is lower hemicontinuous along line segments, if and only if the function $\xi \mapsto\langle A \xi, u-v\rangle$ is lower semicontinuous for all $u, v \in \mathbb{K}$ on the line segments $[u, v]$.

Definition 5.6. A mapping $A: \mathbb{K} \rightarrow X^{*}$ is hemicontinuous in the sense of Fan (Fhemicontinuous) if and only if for all $v \in \mathbb{K}$ the function $u \mapsto\langle A u, u-v\rangle$ is weakly lower semicontinuous on $\mathbb{K}$.

Let us recall that in the Hilbert space $L^{2}\left([0, T], \mathbb{R}^{k}\right)$

$$
\ll \phi, y \gg=\int_{0}^{T}\langle\phi(t), y(t)\rangle d t
$$

is its duality mapping, where $\phi \in\left(L^{2}\left([0, T], \mathbb{R}^{k}\right)\right)^{*}=L^{2}\left([0, T], \mathbb{R}^{k}\right)$ and $y \in L^{2}\left([0, T], \mathbb{R}^{k}\right)$. We are able to show the following existence result.

Theorem 5.7. Let one set

$$
\begin{aligned}
& A=\left[-\frac{\partial v_{i}\left(x^{*}\right)}{\partial x_{i j}}\right]_{\substack{i=1, \ldots, m \\
j=1, \ldots, n}}, \\
& A: L^{2}\left([0, T], \mathbb{R}^{m n}\right) \longrightarrow L^{2}\left([0, T], \mathbb{R}^{m n}\right), \\
& u=\left(x_{i j}\right)_{\substack{i=1, \ldots, m \\
j=1, \ldots, n}} \\
& \mathbb{K}=\left\{x \in L^{2}\left([0, T], \mathbb{R}^{m n}\right):\right. \\
& \underline{x}_{i j}(t) \leq x_{i j}(t) \leq \bar{x}_{i j}(t), \forall i=1, \ldots, m, \forall j=1, \ldots, n \text {, a.e. in }[0, T], \\
& \sum_{j=1}^{n} x_{i j}(t) \leq p_{i}(t), \forall i=1, \ldots, m \text {, a.e. in }[0, T], \\
& \left.\sum_{i=1}^{m} x_{i j}(t) \leq q_{j}(t), \forall j=1, \ldots, n \text {, a.e. in }[0, T]\right\} \text {. }
\end{aligned}
$$

If $A$ is B-pseudomonotone or F-hemicontinuous, or assuming that $A$ is K-pseudomonotone and lower hemicontinuous along line segments, then the variational inequality

$$
\ll A x^{*}, x-x^{*} \gg \geq 0, \quad \forall x \in \mathbb{K},
$$

admits a solution. 
Proof. Let us note that $\mathbb{K}$ is clearly a nonempty, closed, convex, and bounded subset of $L^{2}\left([0, T], \mathbb{R}^{m n}\right)$, and, consequently, it is a weakly compact subset of $L^{2}\left([0, T], \mathbb{R}^{m n}\right)$. Then, the claim is achieved by applying Theorems 2.6 and 2.7 and Corollary 3.7 in [24].

\section{Regularity Results}

In the following, we want to establish conditions under which the solutions to the dynamic oligopolistic market problem with both production and demand excesses are continuous with respect to time.

\subsection{Set Convergence}

Let us remember the classical notion of convergence for subsets of a given metric space $(X, d)$, which was introduced in the 1950s by Kuratowski (see [25], see also [26, 27]).

Let $\left\{K_{n}\right\}_{n \in \mathbb{N}}$ be a sequence of subsets of $X$. Let us remember that

$$
\begin{aligned}
& d-{\varliminf_{n}}_{n} K_{n}=\left\{x \in X: \exists\left\{x_{n}\right\}_{n \in \mathbb{N}} \text { eventually in } K_{n} \text { such that } x_{n} \stackrel{d}{\longrightarrow} x\right\}, \\
& d-\varlimsup_{n} K_{n}=\left\{x \in X: \exists\left\{x_{n}\right\}_{n \in \mathbb{N}} \text { frequently in } K_{n} \text { such that } x_{n} \stackrel{d}{\longrightarrow} x\right\},
\end{aligned}
$$

where eventually means that there exists $\delta \in \mathbb{N}$ such that $x_{n} \in K_{n}$ for any $n \geq \delta$, and frequently means that there exists an infinite subset $N \subseteq \mathbb{N}$ such that $x_{n} \in K_{n}$ for any $n \in N$ (in this last case, according to the notation given above, we also write that there exists a subsequence $\left\{x_{k_{n}}\right\}_{n \in \mathbb{N}} \subseteq\left\{x_{n}\right\}_{n \in \mathbb{N}}$ such that $\left.x_{k_{n}} \in K_{k_{n}}\right)$.

Now, we are able to recall the Kuratowski's convergence of sets.

Definition 6.1. We say that the sequence $\left\{K_{n}\right\}_{n \in \mathbb{N}}$ converges to some subset $K \subseteq X$ in Kuratowski's sense, and we briefly write $K_{n} \rightarrow K$, if and only if $d-\underline{\lim }_{n} K_{n}=d-\varlimsup_{n} K_{n}=K$.

Thus, in order to verify that $K_{n} \rightarrow K$, it suffices to check that

(i) $K \subset d-\underline{\lim }_{n} K_{n}$, that is, for any $x \in K$ there exists a sequence $\left\{x_{n}\right\}_{n \in \mathbb{N}}$ eventually in $K_{n}$ such that $x_{n} \stackrel{d}{\rightarrow} x$,

(ii) $d-\varlimsup_{n} K_{n} \subseteq K$, that is, for any sequence $\left\{x_{n}\right\}_{n \in \mathbb{N}}$ frequently in $K_{n}$ such that $x_{n} \stackrel{d}{\rightarrow} x$ for some $x \in S$, then $x \in K$. follows.

We observe that the set convergence in Kuratowski's sense can also be expressed as

Remark 6.2. Let $(X, d)$ be a metric space and $K$ a nonempty, closed, and convex subset of $X$. A sequence of nonempty, closed and convex sets $K_{n}$ of $X$ converges to $K$ in Kuratowski's sense, as $n \rightarrow+\infty$, that is, $K_{n} \rightarrow K$, if and only if

(K1) for any $x \in K$, there exists a sequence $\left\{x_{n}\right\}_{n \in \mathbb{N}}$ converging to $x \in X$ such that $x_{n}$ lies in $K_{n}$ for all $n \in \mathbb{N}$,

(K2) for any subsequence $\left\{x_{n}\right\}_{n \in \mathbb{N}}$ converging to $x \in X$ such that $x_{n}$ lies in $K_{n}$, for all $n \in \mathbb{N}$, then the limit $x$ belongs to $K$. 
The following lemma, that now we prove, assures that the feasible set $K$ of the dynamic oligopolistic market problem in the presence of both production and demand excesses satisfies the property of the set convergence in Kuratowski's sense.

Lemma 6.3. Let $\underline{x}, \bar{x} \in C^{0}\left([0, T], \mathbb{R}_{+}^{m n}\right), p \in C^{0}\left([0, T], \mathbb{R}_{+}^{m}\right), q \in C^{0}\left([0, T], \mathbb{R}_{+}^{n}\right)$, and let $\left\{t_{k}\right\}_{k \in \mathbb{N}}$ be a sequence such that $t_{k} \in[0, T]$, for all $k \in \mathbb{N}$, and $t_{k} \rightarrow t$, with $t \in[0, T]$, as $k \rightarrow+\infty$. Then the sequence of sets

$$
\begin{aligned}
\mathbb{K}\left(t_{k}\right)=\{ & x\left(t_{k}\right) \in \mathbb{R}^{m n}: \underline{x}_{i j}\left(t_{k}\right) \leq x_{i j}\left(t_{k}\right) \leq \bar{x}_{i j}\left(t_{k}\right), \forall i=1, \ldots, m, \forall j=1, \ldots, n, \\
& \sum_{j=1}^{n} x_{i j}\left(t_{k}\right) \leq p_{i}\left(t_{k}\right), \forall i=1, \ldots, m \\
& \left.\sum_{i=1}^{m} x_{i j}\left(t_{k}\right) \leq q_{j}\left(t_{k}\right), \forall j=1, \ldots, n\right\}
\end{aligned}
$$

for all $k \in \mathbb{N}$, converges to

$$
\begin{aligned}
\mathbb{K}(t)=\{ & x(t) \in \mathbb{R}^{m n}: \underline{x}_{i j}(t) \leq x_{i j}(t) \leq \bar{x}_{i j}(t), \forall i=1, \ldots, m, \forall j=1, \ldots, n, \\
& \sum_{j=1}^{n} x_{i j}(t) \leq p_{i}(t), \forall i=1, \ldots, m, \\
& \left.\sum_{i=1}^{m} x_{i j}(t) \leq q_{j}(t), \forall j=1, \ldots, n\right\},
\end{aligned}
$$

as $k \rightarrow+\infty$, in Kuratowski's sense.

Proof. In the first part, we prove the condition (K1). Let $\left\{t_{k}\right\}_{k \in \mathbb{N}}$ be a sequence such that $t_{k} \in$ $[0, T]$, for all $k \in \mathbb{N}$, and $t_{k} \rightarrow t$, with $t \in[0, T]$, as $k \rightarrow+\infty$. By virtue of the continuity of $\underline{x}$, $\bar{x}, p, q$, it follows that $\underline{x}\left(t_{k}\right) \rightarrow \underline{x}(t), \bar{x}\left(t_{k}\right) \rightarrow \bar{x}(t), p\left(t_{k}\right) \rightarrow p(t), q\left(t_{k}\right) \rightarrow q(t)$, as $k \rightarrow+\infty$, respectively. Let $x(t) \in \mathbb{K}(t)$ be fixed and let us note that, for $i=1, \ldots, m$ and $j=1, \ldots, n$, and if

$$
a_{i j}\left(t_{k}\right)=x_{i j}(t)-\underline{x}_{i j}\left(t_{k}\right)+\frac{m p_{i}\left(t_{k}\right)+n q_{j}\left(t_{k}\right)}{m n}-\frac{m p_{i}(t)+n q_{j}(t)}{m n},
$$

it results in

$$
\lim _{k \rightarrow+\infty} a_{i j}\left(t_{k}\right)=x_{i j}(t)-\underline{x}_{i j}(t) \geq 0
$$

As a consequence, there exists an index $v_{1}$ such that for $k>v_{1}$ we get

$$
a_{i j}\left(t_{k}\right) \geq 0, \quad \forall i=1, \ldots, m, \forall j=1, \ldots, n
$$


We remark

$$
\lim _{k \rightarrow+\infty}\left[\frac{1}{m} \sum_{j=1}^{n} q_{j}\left(t_{k}\right)-\frac{1}{m} \sum_{j=1}^{n} q_{j}(t)-\epsilon_{i}(t)\right]=-\epsilon_{i}(t) \leq 0, \quad \forall i=1, \ldots, m,
$$

where $\epsilon$ is the production excess function. Then, there exists an index $v_{2}$ such that for $k>v_{2}$ we have

$$
\frac{1}{m} \sum_{j=1}^{n} q_{j}\left(t_{k}\right)-\frac{1}{m} \sum_{j=1}^{n} q_{j}(t)-\epsilon_{i}(t) \leq 0, \quad \forall i=1, \ldots, m
$$

Moreover, we get

$$
\lim _{k \rightarrow+\infty}\left[\frac{1}{n} \sum_{i=1}^{m} p_{i}\left(t_{k}\right)-\frac{1}{n} \sum_{i=1}^{m} p_{i}(t)-\delta_{j}(t)\right]=-\delta_{j}(t) \leq 0, \quad \forall j=1, \ldots, n,
$$

where $\delta$ is the demand excess function. Hence, there exists an index $v_{3}$ such that for $k>v_{3}$ we have

$$
\frac{1}{n} \sum_{i=1}^{m} p_{i}\left(t_{k}\right)-\frac{1}{n} \sum_{i=1}^{m} p_{i}(t)-\delta_{j}(t) \leq 0, \quad \forall j=1, \ldots, n
$$

As a consequence, we can consider a sequence $\left\{x\left(t_{k}\right)\right\}_{k \in \mathbb{N}}$ such that:

(i) for $k>v=\max \left\{v_{1}, v_{2}, v_{3}\right\}$, for all $i=1, \ldots, m$, for all $j=1, \ldots, n$,

$$
x_{i j}\left(t_{k}\right)=\underline{x}_{i j}\left(t_{k}\right)+\min \left\{x_{i j}(t)-\underline{x}_{i j}(t), \bar{x}_{i j}\left(t_{k}\right)-\underline{x}_{i j}\left(t_{k}\right), a_{i j}\left(t_{k}\right)\right\},
$$

(ii) for $k \leq v$, for all $i=1, \ldots, m$, for all $j=1, \ldots, n$,

$$
x_{i j}\left(t_{k}\right)=P_{\mathbb{K}\left(t_{k}\right)} x_{i j}(t),
$$

where $P_{\mathbb{K}\left(t_{k}\right)}(\cdot)$ denotes the Hilbertian projection on $\mathbb{K}\left(t_{k}\right)$.

Obviously if $k \leq v$, for (6.12) we get $x\left(t_{k}\right) \in \mathbb{K}\left(t_{k}\right)$. Instead, for $k>v$, since for (6.6), $\min \left\{x_{i j}(t)-\underline{x}_{i j}(t), \bar{x}_{i j}\left(t_{k}\right)-\underline{x}_{i j}\left(t_{k}\right), a_{i j}\left(t_{k}\right)\right\} \geq 0$, for all $i=1, \ldots, m$, for all $j=1, \ldots, n$, we obtain

$$
\underline{x}_{i j}\left(t_{k}\right) \leq x_{i j}\left(t_{k}\right), \quad \forall i=1, \ldots, m, \forall j=1, \ldots, n
$$

Moreover, since $\min \left\{x_{i j}(t)-\underline{x}_{i j}(t), \bar{x}_{i j}\left(t_{k}\right)-\underline{x}_{i j}\left(t_{k}\right), a_{i j}\left(t_{k}\right)\right\} \leq \bar{x}_{i j}\left(t_{k}\right)-\underline{x}_{i j}\left(t_{k}\right)$, for all $i=$ $1, \ldots, m$, for all $j=1, \ldots, n$, we have

$$
x_{i j}\left(t_{k}\right) \leq \bar{x}_{i j}\left(t_{k}\right), \quad \forall i=1, \ldots, m, \forall j=1, \ldots, n
$$


Now, being

$$
\begin{aligned}
& \min \left\{x_{i j}(t)-\underline{x}_{i j}(t), \bar{x}_{i j}\left(t_{k}\right)-\underline{x}_{i j}\left(t_{k}\right), a_{i j}\left(t_{k}\right)\right\} \\
& \leq a_{i j}\left(t_{k}\right) \\
&=x_{i j}(t)-\underline{x}_{i j}\left(t_{k}\right)+\frac{m p_{i}\left(t_{k}\right)+n q_{j}\left(t_{k}\right)}{m n}-\frac{m p_{i}(t)+n q_{j}(t)}{m n} \\
& \forall i=1, \ldots, m, \forall j=1, \ldots, n,
\end{aligned}
$$

it results in

$$
x_{i j}\left(t_{k}\right) \leq x_{i j}(t)+\frac{m p_{i}\left(t_{k}\right)+n q_{j}\left(t_{k}\right)}{m n}-\frac{m p_{i}(t)+n q_{j}(t)}{m n}, \quad \forall i=1, \ldots, m, \forall j=1, \ldots, n .
$$

Then, taking into account (6.8), we get

$$
\begin{aligned}
\sum_{j=1}^{n} x_{i j}\left(t_{k}\right) & \leq \sum_{j=1}^{n} x_{i j}(t)+p_{i}\left(t_{k}\right)+\frac{1}{m} \sum_{j=1}^{n} q_{j}\left(t_{k}\right)-p_{i}(t)-\frac{1}{m} \sum_{j=1}^{n} q_{j}(t) \\
& \leq \sum_{j=1}^{n} x_{i j}(t)+p_{i}\left(t_{k}\right)-p_{i}(t)+\epsilon_{i}(t) \\
& =\sum_{j=1}^{n} x_{i j}(t)+p_{i}\left(t_{k}\right)-\sum_{j=1}^{n} x_{i j}(t)-\epsilon_{i}(t)+\epsilon_{i}(t) \\
& =p_{i}\left(t_{k}\right), \quad \forall i=1, \ldots, m,
\end{aligned}
$$

and, making use of (6.10), we obtain

$$
\begin{aligned}
\sum_{i=1}^{m} x_{i j}\left(t_{k}\right) & \leq \sum_{i=1}^{m} x_{i j}(t)+\frac{1}{n} \sum_{i=1}^{m} p_{i}\left(t_{k}\right)+q_{j}\left(t_{k}\right)-\frac{1}{n} \sum_{i=1}^{m} p_{i}(t)+q_{j}(t) \\
& \leq \sum_{i=1}^{m} x_{i j}(t)+q_{j}\left(t_{k}\right)-q_{j}(t)+\delta_{j}(t) \\
& =\sum_{i=1}^{m} x_{i j}(t)+q_{j}\left(t_{k}\right)-\sum_{i=1}^{m} x_{i j}(t)-\delta_{j}(t)+\delta_{j}(t) \\
& =q_{j}\left(t_{k}\right), \quad \forall j=1, \ldots, n .
\end{aligned}
$$


Hence $x\left(t_{k}\right) \in \mathbb{K}\left(t_{k}\right)$, for all $k \in \mathbb{N}$, and it results in

$$
\begin{aligned}
\lim _{k \rightarrow+\infty} x_{i j}\left(t_{k}\right) & =\underline{x}_{i j}(t)+\min \left\{x_{i j}(t)-\underline{x}_{i j}(t), \bar{x}_{i j}(t)-\underline{x}_{i j}(t), x_{i j}(t)-\underline{x}_{i j}(t)\right\} \\
& =\underline{x}_{i j}(t)+x_{i j}(t)-\underline{x}_{i j}(t) \\
& =x_{i j}(t) .
\end{aligned}
$$

Then, the proof of the condition (K1) is completed.

Now let us prove condition (K2). Let $\left\{t_{k}\right\}_{k \in \mathbb{N}}$ be a sequence such that $t_{k} \in[0, T]$, for all $k \in \mathbb{N}$, and $t_{k} \rightarrow t$, with $t \in[0, T]$, as $k \rightarrow+\infty$. Let $\left\{x\left(t_{k}\right)\right\}_{k \in \mathbb{N}}$ be a sequence, such that $x\left(t_{k}\right) \in \mathbb{K}\left(t_{k}\right)$, for all $k \in \mathbb{N}$, and converging to $x(t)$, as $k \rightarrow+\infty$. We need to prove that $x(t) \in \mathbb{K}(t)$.

Since $x\left(t_{k}\right) \in \mathbb{K}\left(t_{k}\right)$, for all $k \in \mathbb{N}$, it results in

$$
\begin{gathered}
\underline{x}_{i j}\left(t_{k}\right) \leq x_{i j}\left(t_{k}\right) \leq \bar{x}_{i j}\left(t_{k}\right), \quad \forall i=1, \ldots, m, \forall j=1, \ldots, n, \forall k \in \mathbb{N}, \\
\quad \sum_{j=1}^{n} x_{i j}\left(t_{k}\right) \leq p_{i}\left(t_{k}\right), \quad \forall i=1, \ldots, m, \forall k \in \mathbb{N}, \\
\quad \sum_{i=1}^{m} x_{i j}\left(t_{k}\right) \leq q_{j}\left(t_{k}\right), \quad \forall j=1, \ldots, n, \forall k \in \mathbb{N} .
\end{gathered}
$$

Passing to the limit as $n \rightarrow+\infty$ and taking into account the continuity assumption on the functions $\underline{x}, \bar{x}, p, q$, we obtain

$$
\begin{gathered}
\underline{x}_{i j}(t) \leq x_{i j}(t) \leq \bar{x}_{i j}(t), \quad \forall i=1, \ldots, m, \forall j=1, \ldots, n, \\
\sum_{j=1}^{n} x_{i j}(t) \leq p_{i}(t), \quad \forall i=1, \ldots, m, \\
\sum_{i=1}^{n} x_{i j}(t) \leq q_{j}(t), \quad \forall j=1, \ldots, n .
\end{gathered}
$$

As a consequence $x(t) \in \mathbb{K}(t)$, and, hence, the condition (K2) is achieved.

\subsection{Continuity Theorems for Equilibrium Solutions}

In order to show the continuity result for the dynamic oligopolistic market equilibrium solution in presence of both production and demand excesses, we present the following result (see e.g., [2, Corollary 3.1]). 


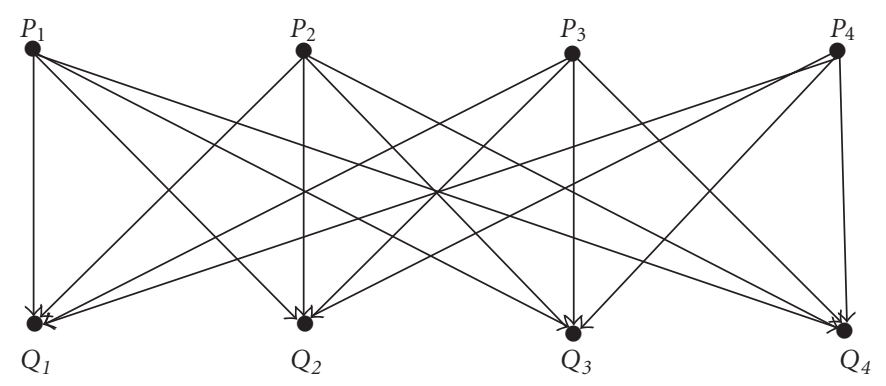

Figure 1: Network structure of the numerical dynamic spatial oligopoly problem.

Proposition 6.4. The evolutionary variational inequality (2.20) is equivalent to the point-to-point variational inequality:

$$
\sum_{i=1}^{m} \sum_{j=1}^{n}-\frac{\partial v_{i}\left(t, x^{*}(t)\right)}{\partial x_{i j}}\left(x_{i j}(t)-x_{i j}^{*}(t)\right) \geq 0, \quad \forall x(t) \in \mathbb{K}(t) \text {, a.e. in }[0, T],
$$

where

$$
\begin{aligned}
\mathbb{K}(t)=\{ & x(t) \in \mathbb{R}^{m n}: \underline{x}_{i j}(t) \leq x_{i j}(t) \leq \bar{x}_{i j}(t), \forall i=1, \ldots, m, \forall j=1, \ldots, n, \\
& \sum_{j=1}^{n} x_{i j}(t) \leq p_{i}(t), \forall i=1, \ldots, m, \\
& \left.\sum_{i=1}^{m} x_{i j}(t) \leq q_{j}(t), \forall j=1, \ldots, n\right\} .
\end{aligned}
$$

The continuity of solutions to evolutionary variational inequalities with respect to time under the only assumption of continuity on the data has been proved in several papers (see for instance [8-12]). Now, in our case, by applying Theorem 4.2 in [12] and taking into account Lemma 6.3, we obtain the following result.

Theorem 6.5. Let one assume that the production function $p$, the demand function $q$, and the capacity constraints $\underline{x}$ and $\bar{x}$ are continuous on $[0, T]$. Moreover, let one assume that the function $-\nabla_{D} v$ is a strictly pseudomonotone and continuous on $[0, T]$. Then the unique dynamic market equilibrium distribution in presence of both production and demand excesses $x^{*} \in \mathbb{K}$ is continuous on $[0, T]$.

\section{Numerical Example}

Let us present a numerical example about the dynamic oligopolistic market equilibrium problem in presence of both production and demand excesses. 
Let us consider four firms and four demand markets, as in Figure 1. Let $\underline{x}, \bar{x} \in$ $L^{2}\left([0,1], \mathbb{R}_{+}^{16}\right)$ be the capacity constraints such that, a.e. in $[0,1]$,

$$
\begin{aligned}
& \underline{x}(t)=\left(\begin{array}{cccc}
\frac{1}{2} t & 0 & \frac{1}{100} t & \frac{1}{2} t \\
t & \frac{1}{4} t & 0 & \frac{1}{10} t \\
\frac{3}{100} t & \frac{1}{4} t & \frac{1}{10} t & \frac{1}{4} t \\
\frac{4}{7} t & \frac{2}{7} t & \frac{1}{10} t & 0
\end{array}\right), \\
& \bar{x}(t)=\left(\begin{array}{cccc}
t & 2 t & t & 4 t \\
2 t & \frac{3}{2} t & \frac{10}{11} t & \frac{5}{6} t \\
t & \frac{3}{4} t & 3 t & \frac{3}{4} t \\
\frac{6}{7} t & \frac{6}{7} t & \frac{3}{7} t & \frac{1}{2} t
\end{array}\right) .
\end{aligned}
$$

Let $p \in L^{2}\left([0,1], \mathbb{R}_{+}^{4}\right)$ be the production function such that, a.e. in $[0,1]$,

$$
p(t)=\left(\begin{array}{c}
5 t \\
7 t \\
9 t \\
11 t
\end{array}\right),
$$

and let $q \in L^{2}\left([0,1], \mathbb{R}_{+}^{4}\right)$ be the demand function such that, a.e. in $[0,1]$,

$$
q(t)=\left(\begin{array}{c}
6 t \\
8 t \\
10 t \\
12 t
\end{array}\right)
$$


As a consequence, the feasible set is

$$
\begin{aligned}
\mathbb{K}=\{ & x \in L^{2}\left([0,1], \mathbb{R}_{+}^{16}\right): \\
& \underline{x}_{i j}(t) \leq x_{i j}(t) \leq \bar{x}_{i j}(t), \forall i=1, \ldots, 4, \forall j=1, \ldots, 4, \text { a.e. in }[0,1], \\
& \sum_{j=1}^{4} x_{i j}(t) \leq p_{i}(t), \forall i=1, \ldots, 4, \text { a.e. in }[0,1], \\
& \left.\sum_{i=1}^{4} x_{i j}(t) \leq q_{j}(t), \forall j=1, \ldots, 4, \text { a.e. in }[0,1]\right\} .
\end{aligned}
$$

Let us consider the profit function $v \in L^{2}\left([0,1] \times L^{2}\left([0,1], \mathbb{R}_{+}^{4 \times 4}\right), \mathbb{R}^{4}\right)$ defined by

$$
\begin{aligned}
v_{1}(t, x(t))= & -4 x_{11}^{2}(t)-6 x_{12}^{2}(t)-2 x_{13}^{2}(t)-4 x_{14}^{2}(t)-2 x_{11}(t) x_{13}(t)-2 x_{12}(t) x_{14}(t) \\
& +3 t x_{11}(t)+6 t x_{12}(t)+t x_{13}(t)+5 t x_{14}(t), \\
v_{2}(t, x(t))= & -2 x_{21}^{2}(t)-5 x_{22}^{2}(t)-6 x_{23}^{2}(t)-2 x_{24}^{2}(t)-2 x_{21}(t) x_{23}(t)-2 x_{22}(t) x_{31}(t) \\
& -2 x_{24}(t) x_{33}(t)+6 t x_{21}(t)+5 t x_{22}(t)+4 t x_{23}(t)+t x_{24}(t), \\
v_{3}(t, x(t))= & -3 x_{31}^{2}(t)-4 x_{32}^{2}(t)-2 x_{33}^{2}(t)-2 x_{34}^{2}(t)-2 x_{22}(t) x_{31}(t)-2 x_{24}(t) x_{33}(t) \\
& -2 x_{32}(t) x_{34}(t)+2 t x_{31}(t)+5 t x_{32}(t)+t x_{33}(t)+3 t x_{34}(t), \\
v_{4}(t, x(t))= & -4 x_{41}^{2}(t)-4 x_{42}^{2}(t)-2 x_{43}^{2}(t)-2 x_{44}^{2}(t)-2 x_{41}(t) x_{43}(t)-2 x_{42}(t) x_{44}(t) \\
& +6 t x_{41}(t)+5 t x_{42}(t)+2 t x_{43}(t)+2 t x_{44}(t) .
\end{aligned}
$$

Then, the operator $\nabla_{D} v \in L^{2}\left([0,1] \times L^{2}\left([0,1], \mathbb{R}_{+}^{4 \times 4}\right), \mathbb{R}^{4 \times 4}\right)$ is given by

$$
\begin{aligned}
& \nabla_{D} v(t, x(t)) \\
& =\left(\begin{array}{llll}
-8 x_{11}(t)-2 x_{13}(t)+3 t & -12 x_{12}(t)-2 x_{14}(t)+6 t & -4 x_{13}(t)-2 x_{11}(t)+t & -8 x_{14}(t)-2 x_{12}(t)+5 t \\
-4 x_{21}(t)-2 x_{23}(t)+6 t & -10 x_{22}(t)-2 x_{31}(t)+5 t & -12 x_{23}(t)-2 x_{21}(t)+4 t & -4 x_{24}(t)-2 x_{33}(t)+t \\
-6 x_{31}(t)-2 x_{22}(t)+2 t & -8 x_{32}(t)-2 x_{34}(t)+5 t & -4 x_{33}(t)-2 x_{24}(t)+t & -4 x_{34}(t)-2 x_{32}(t)+3 t \\
-8 x_{41}(t)-2 x_{43}(t)+6 t & -8 x_{42}(t)-2 x_{44}(t)+5 t & -4 x_{43}(t)-2 x_{41}(t)+2 t & -4 x_{44}(t)-2 x_{42}(t)+2 t
\end{array}\right) .
\end{aligned}
$$


Now, we verify that $-\nabla_{D} v$ is a strongly monotone operator, in fact

$$
\begin{aligned}
\left\langle-\nabla_{D} v(x)+\nabla_{D} v(y), x-y\right\rangle= & \left\{8\left[x_{11}-y_{11}\right]+2\left[x_{13}-y_{13}\right]\right\}\left[x_{11}-y_{11}\right] \\
& +\left\{12\left[x_{12}-y_{12}\right]+2\left[x_{14}-y_{14}\right]\right\}\left[x_{12}-y_{12}\right] \\
& +\left\{4\left[x_{13}-y_{13}\right]+2\left[x_{11}-y_{11}\right]\right\}\left[x_{13}-y_{13}\right] \\
& +\left\{8\left[x_{14}-y_{14}\right]+2\left[x_{12}-y_{12}\right]\right\}\left[x_{14}-y_{14}\right] \\
& +\left\{4\left[x_{21}-y_{21}\right]+2\left[x_{23}-y_{23}\right]\right\}\left[x_{21}-y_{21}\right] \\
& +\left\{10\left[x_{22}-y_{22}\right]+2\left[x_{31}-y_{31}\right]\right\}\left[x_{22}-y_{22}\right] \\
& +\left\{12\left[x_{23}-y_{23}\right]+2\left(x_{21}-y_{21}\right]\right\}\left[x_{23}-y_{23}\right] \\
& +\left\{4\left[x_{24}-y_{24}\right]+2\left[x_{33}-y_{33}\right]\right\}\left[x_{24}-y_{24}\right] \\
& +\left\{6\left[x_{31}-y_{31}\right]+2\left[x_{22}-y_{22}\right]\right\}\left[x_{31}-y_{31}\right] \\
& +\left\{8\left[x_{32}-y_{32}\right]+2\left[x_{34}-y_{34}\right]\right\}\left[x_{32}-y_{32}\right] \\
& +\left\{4\left[x_{33}-y_{33}\right]+2\left[x_{24}-y_{24}\right]\right\}\left[x_{33}-y_{33}\right] \\
& +\left\{4\left[x_{34}-y_{34}\right]+2\left[x_{32}-y_{32}\right]\right\}\left[x_{34}-y_{34}\right] \\
& +\left\{8\left[x_{41}-y_{41}\right]+2\left[x_{43}-y_{43}\right]\right\}\left[x_{41}-y_{41}\right] \\
& +\left\{8\left[x_{42}-y_{42}\right]+2\left[x_{44}-y_{44}\right]\right\}\left[x_{42}-y_{42}\right] \\
& +\left\{4\left[x_{43}-y_{43}\right]+2\left[x_{41}-y_{41}\right]\right\}\left[x_{43}-y_{43}\right] \\
& +\left\{4\left[x_{44}-y_{44}\right]+2\left[x_{42}-y_{42}\right]\right\}\left[x_{44}-y_{44}\right] \\
\geq & 3\|x-y\|_{4}^{2} \times 4
\end{aligned}
$$

The dynamic oligopolistic market equilibrium distribution in presence of excesses is the solution to the evolutionary variational inequality:

$$
\int_{0}^{1} \sum_{i=1}^{4} \sum_{j=1}^{4}-\frac{\partial v_{i}\left(t, x^{*}(t)\right)}{\partial x_{i j}}\left(x_{i j}(t)-x_{i j}^{*}(t)\right) d t \geq 0, \quad \forall x \in \mathbb{K} .
$$

In order to compute the solution to (7.8) we make use of the direct method (see [2830]). We consider the following system:

$$
\begin{array}{ccc}
8 x_{11}^{*}(t)+2 x_{13}^{*}(t)-3 t=0, & 12 x_{12}^{*}(t)+2 x_{14}^{*}(t)-6 t=0, & 4 x_{13}^{*}(t)+2 x_{11}^{*}(t)-t=0, \\
8 x_{14}^{*}(t)+2 x_{12}^{*}(t)-5 t=0, & 4 x_{21}^{*}(t)+2 x_{23}^{*}(t)-6 t=0, & 10 x_{22}^{*}(t)+2 x_{31}^{*}(t)-5 t=0, \\
12 x_{23}^{*}(t)+2 x_{21}^{*}(t)-4 t=0, & 4 x_{24}^{*}(t)+2 x_{33}^{*}(t)-t=0, & 6 x_{31}^{*}(t)+2 x_{22}^{*}(t)-2 t=0, \\
8 x_{32}^{*}(t)+2 x_{34}^{*}(t)-5 t=0, & 4 x_{33}^{*}(t)+2 x_{24}^{*}(t)-t=0, & 4 x_{34}^{*}(t)+2 x_{32}^{*}(t)-3 t=0, \\
8 x_{41}^{*}(t)+2 x_{43}^{*}(t)-6 t=0, & 8 x_{42}^{*}(t)+2 x_{44}^{*}(t)-5 t=0, & 4 x_{43}^{*}(t)+2 x_{41}^{*}(t)-2 t=0,
\end{array}
$$


Abstract and Applied Analysis

$$
\begin{gathered}
4 x_{44}^{*}(t)+2 x_{42}^{*}(t)-2 t=0, \\
\underline{x}_{i j}(t) \leq x_{i j}(t) \leq \bar{x}_{i j}(t), \quad \forall i=1, \ldots, 4, \forall j=1, \ldots, 4, \\
\sum_{j=1}^{n} x_{i j}(t) \leq p_{i}(t), \quad \forall i=1, \ldots, 4, \\
\sum_{i=1}^{m} x_{i j}(t) \leq q_{j}(t), \quad \forall j=1, \ldots, 4,
\end{gathered}
$$

and we get the following solution, a.e. in $[0,1]$ :

$$
x^{*}(t)=\left(\begin{array}{cccc}
\frac{5}{14} t & \frac{19}{46} t & \frac{1}{14} t & \frac{12}{23} t \\
\frac{16}{11} t & \frac{13}{28} t & \frac{1}{11} t & \frac{1}{6} t \\
\frac{5}{28} t & \frac{1}{2} t & \frac{1}{6} t & \frac{1}{2} t \\
\frac{5}{7} t & \frac{4}{7} t & \frac{1}{7} t & \frac{3}{14} t
\end{array}\right) .
$$

It is easy to prove that $x^{*}$ belongs to the constraint set $\mathbb{K}$, then it is the equilibrium solution.

Now, we are able to compute the production excess of each firm and the demand excess of each demand market

$$
\begin{aligned}
& \epsilon_{1}(t)=p_{1}(t)-\sum_{j=1}^{4} x_{1 j}^{*}(t)=5 t-\frac{439}{322} t=\frac{1171}{322} t, \quad \text { a.e. in }[0,1], \\
& \epsilon_{2}(t)=p_{2}(t)-\sum_{j=1}^{4} x_{2 j}^{*}(t)=7 t-\frac{2011}{924} t=\frac{4457}{924} t, \quad \text { a.e. in }[0,1], \\
& \epsilon_{3}(t)=p_{3}(t)-\sum_{j=1}^{4} x_{3 j}^{*}(t)=9 t-\frac{113}{84} t=\frac{643}{84} t, \quad \text { a.e. in }[0,1], \\
& \epsilon_{4}(t)=p_{4}(t)-\sum_{j=1}^{4} x_{3 j}^{*}(t)=11 t-\frac{23}{14} t=\frac{131}{14} t, \quad \text { a.e. in }[0,1], \\
& \delta_{1}(t)=q_{1}(t)-\sum_{i=1}^{4} x_{i 1}^{*}(t)=6 t-\frac{119}{44} t=\frac{145}{44} t, \quad \text { a.e. in }[0,1], \\
& \delta_{2}(t)=q_{2}(t)-\sum_{i=1}^{4} x_{i 2}^{*}(t)=8 t-\frac{1255}{644} t=\frac{3897}{644} t, \quad \text { a.e. in }[0,1],
\end{aligned}
$$




$$
\begin{aligned}
& \delta_{3}(t)=q_{3}(t)-\sum_{i=1}^{4} x_{i 3}^{*}(t)=10 t-\frac{109}{231} t=\frac{2201}{231} t, \quad \text { a.e. in }[0,1] \\
& \delta_{4}(t)=q_{4}(t)-\sum_{i=1}^{4} x_{i 4}^{*}(t)=12 t-\frac{1355}{966} t=\frac{10237}{966} t, \quad \text { a.e. in }[0,1] .
\end{aligned}
$$

Making use of the equilibrium conditions (2.21)-(2.25), we derive:

$$
\begin{aligned}
& \lambda_{i j}^{*}(t)=0, \quad \forall i=1, \ldots, 4, \forall j=1, \ldots, 4, \text { a.e. in }[0,1], \\
& \rho_{i j}^{*}(t)=0, \quad \forall i=1, \ldots, 4, \forall j=1, \ldots, 4, \text { a.e. in }[0,1], \\
& \mu_{i}^{*}(t)=0, \quad \forall i=1, \ldots, 4, \text { a.e. in }[0,1], \\
& v_{j}^{*}(t)=0, \quad \forall j=1, \ldots, 4, \text { a.e. in }[0,1] .
\end{aligned}
$$

\section{Concluding Remarks}

In this paper, we have considered the variational formulation for the dynamic oligopolistic market equilibrium problem in presence of both production and demand excesses. The very general model allows to study all the economic periods that a market can be gone through. In this way, the previous models presented in [2] and in [1] are improved.

The equilibrium conditions are given according to the well-known dynamic CournotNash principle and by means Lagrange multipliers which allow to point out the importance of the excesses in the equilibrium solutions. Making use of the variational formulation, which expresses the equilibrium conditions, the equivalence between the two equilibrium definitions is proved. In particular, the evolutionary variational inequality allows to obtain very important theoretical results for equilibrium solutions. More precisely, under general assumptions, the existence of equilibrium solutions is guaranteed. Moreover, after that the powerful property of the set convergence in Kuratowski's sense has been proved for the constraint set, a continuity result for the equilibrium solution has been obtained.

\section{References}

[1] A. Barbagallo and P. Mauro, "Evolutionary variational formulation for oligopolistic market equilibrium problems with production excesses," Journal of Optimization Theory and Applications, vol. 155, no. 1, 2012.

[2] A. Barbagallo and M.-G. Cojocaru, "Dynamic equilibrium formulation of the oligopolistic market problem," Mathematical and Computer Modelling, vol. 49, no. 5-6, pp. 966-976, 2009.

[3] A. Cournot, Researches Into The Mathematical Principles Of The Theory Of Wealth, MacMillan, London, UK, 1838.

[4] J. F. Nash Jr., "Equilibrium points in n-person games," Proceedings of the National Academy of Sciences of the United States of America, vol. 36, pp. 48-49, 1950.

[5] J. Nash, "Non-cooperative games," Annals of Mathematics. Second Series, vol. 54, pp. 286-295, 1951.

[6] M. J. Beckmann and J. P. Wallace, "Continuous lags and the stability of market equilibrium," Economica, New Series 36, pp. 58-68, 1969.

[7] A. Barbagallo and A. Maugeri, "Memory term for dynamic oligopolistic market equilibrium problem," Aplimat, Journal of Applied Mathematics, vol. 3, pp. 13-23, 2010. 
[8] A. Barbagallo, "Regularity results for time-dependent variational and quasi-variational inequalities and application to the calculation of dynamic traffic network," Mathematical Models $\mathcal{E}$ Methods in Applied Sciences, vol. 17, no. 2, pp. 277-304, 2007.

[9] A. Barbagallo, "Regularity results for evolutionary nonlinear variational and quasi-variational inequalities with applications to dynamic equilibrium problems," Journal of Global Optimization, vol. 40, no. 1-3, pp. 29-39, 2008.

[10] A. Barbagallo, "Existence and regularity of solutions to nonlinear degenerate evolutionary variational inequalities with applications to dynamic network equilibrium problems," Applied Mathematics and Computation, vol. 208, no. 1, pp. 1-13, 2009.

[11] A. Barbagallo, "On the regularity of retarded equilibrium in time-dependent traffic equilibrium problems," Nonlinear Analysis, vol. 71, no. 12, pp. e2406-e2417, 2009.

[12] A. Barbagallo and M.-G. Cojocaru, "Continuity of solutions for parametric variational inequalities in Banach space," Journal of Mathematical Analysis and Applications, vol. 351, no. 2, pp. 707-720, 2009.

[13] A. Barbagallo and A. Maugeri, "Duality theory for the dynamic oligopolistic market equilibrium problem," Optimization, vol. 60, no. 1-2, pp. 29-52, 2011.

[14] J. M. Borwein and A. S. Lewis, "Partially finite convex programming. I. Quasi relative interiors and duality theory," Mathematical Programming, vol. 57, no. 1, Ser. B, pp. 15-48, 1992.

[15] A. Maugeri and F. Raciti, "Remarks on infinite dimensional duality," Journal of Global Optimization, vol. 46, no. 4, pp. 581-588, 2010.

[16] P. Daniele and S. Giuffrè, "General infinite dimensional duality and applications to evolutionary network equilibrium problems," Optimization Letters, vol. 1, no. 3, pp. 227-243, 2007.

[17] P. Daniele, S. Giuffrè, G. Idone, and A. Maugeri, "Infinite dimensional duality and applications," Mathematische Annalen, vol. 339, no. 1, pp. 221-239, 2007.

[18] P. Daniele, S. Giuffré, and A. Maugeri, "Remarks on general infinite dimensional duality with cone and equality constraints," Communications in Applied Analysis, vol. 13, no. 4, pp. 567-577, 2009.

[19] A. Barbagallo and R. Di Vincenzo, "Lipschitz continuity and duality for dynamic oligopolistic market equilibrium problem with memory term," Journal of Mathematical Analysis and Applications, vol. 382, no. 1 , pp. 231-247, 2011.

[20] A. Barbagallo and P. Mauro, “On solving dynamic oligopolistic market equilibrium problems in presence of excesses," Submitted.

[21] A. Barbagallo, "Advanced results on variational inequality formulation in oligopolistic market equilibrium problem," FILOMAT. In press.

[22] O. Mangasarian, "Pseudo-convex functions," Journal of the Society for Industrial and Applied Mathematics Series A, vol. 3, no. 2, pp. 281-290, 1965.

[23] J. Jahn, Introduction to the Theory of Nonlinear Optimization, Springer, Berlin, Germany, 2nd edition, 1996.

[24] A. Maugeri and F. Raciti, "On existence theorems for monotone and nonmonotone variational inequalities," Journal of Convex Analysis, vol. 16, no. 3-4, pp. 899-911, 2009.

[25] K. Kuratowski, Topology, Academic Press, New York, NY, USA, 1966.

[26] G. Salinetti and R. J.-B. Wets, "On the convergence of sequences of convex sets in finite dimensions," SIAM Review, vol. 21, no. 1, pp. 18-33, 1979.

[27] G. Salinetti and R. J.-B. Wets, "Addendum: on the convergence of convex sets in finite dimensions," SIAM Review, vol. 22, p. 86.

[28] A. Maugeri, "Convex programming, variational inequalities, and applications to the traffic equilibrium problem," Applied Mathematics and Optimization, vol. 16, no. 2, pp. 169-185, 1987.

[29] P. Daniele and A. Maugeri, "The economic model for demand-supply problems," in Equilibrium Problems and Variational Models, P. Daniele, F. Giannessi, and A. Maugeri, Eds., pp. 61-78, Kluwer Academic, Boston, Mass, USA, 2002.

[30] P. Daniele, "Evolutionary variational inequalities and economic models for demand-supply markets," Mathematical Models \& Methods in Applied Sciences, vol. 13, no. 4, pp. 471-489, 2003. 


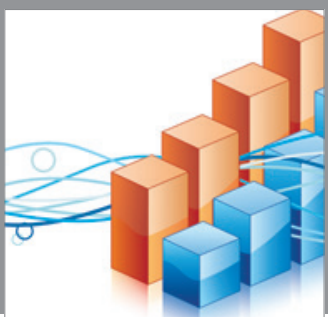

Advances in

Operations Research

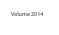

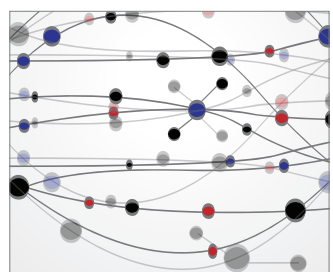

\section{The Scientific} World Journal
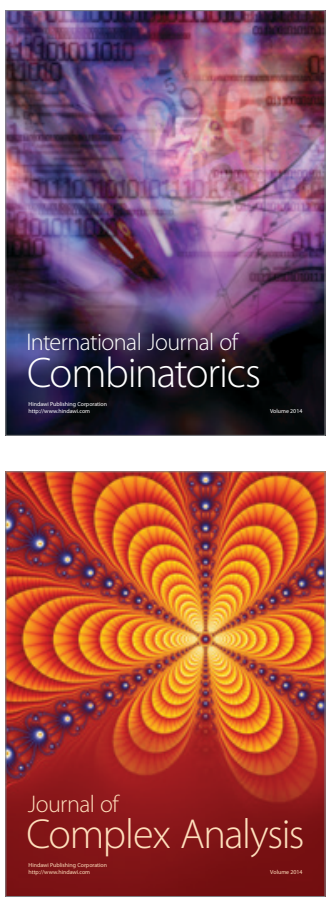

International Journal of

Mathematics and

Mathematical

Sciences
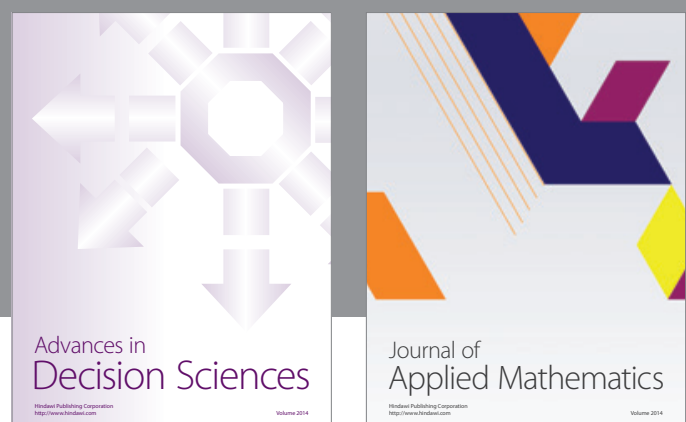

Journal of

Applied Mathematics
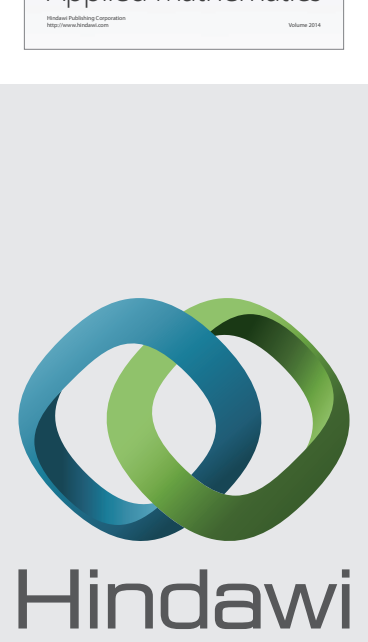

Submit your manuscripts at http://www.hindawi.com
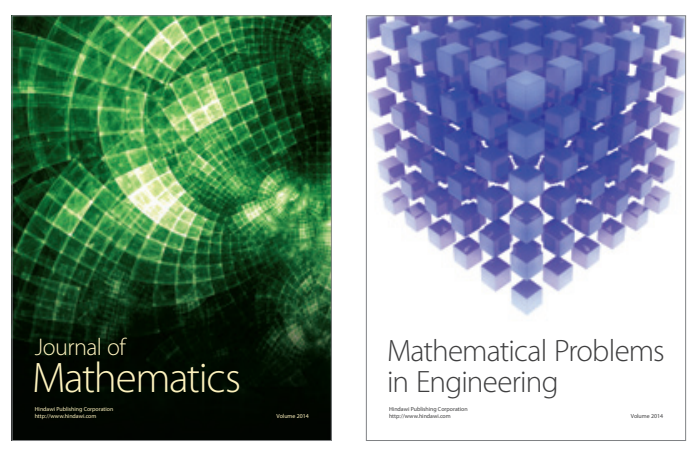

Mathematical Problems in Engineering
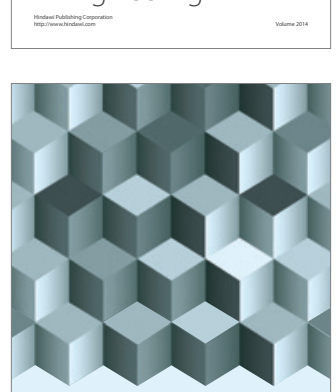

Journal of

Function Spaces
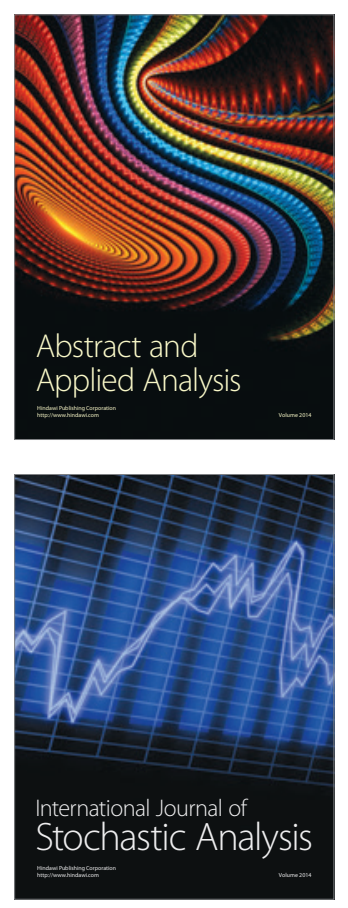

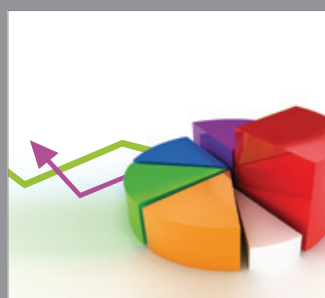

ournal of

Probability and Statistics

Promensencen
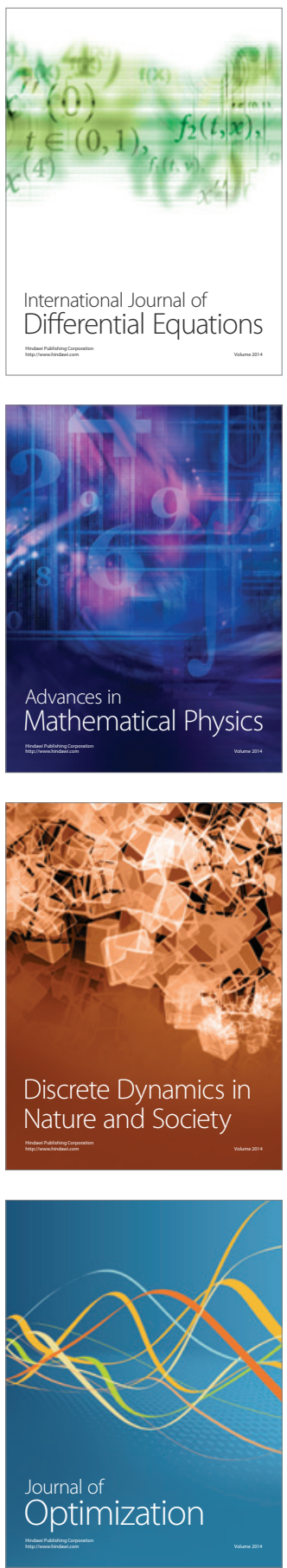